\title{
Recent advances in the design of Choline kinase $\alpha$ inhibitors and the molecular basis of their inhibition
}

\author{
Advances in the design of ChoKa inhibitors \\ Belén Rubio-Ruiz, ${ }^{1,2,3}$ Lucía Serrán-Aguilera, ${ }^{1}$ Ramón Hurtado-Guerrero, ${ }^{4,5,6, *}$ Ana Conejo-García ${ }^{1,3, *}$ \\ ${ }^{1}$ Department of Medicinal and Organic Chemistry, Faculty of Pharmacy, University of Granada, \\ Granada, Spain
}

${ }^{2}$ Pfizer-University of Granada-Andalusian Regional Government Centre for Genomics and Oncological Research (GENYO), Granada, Spain

${ }^{3}$ Biosanitary Institute of Granada (ibs.GRANADA), SAS-University of Granada, Granada, Spain

${ }^{4}$ Institute for Biocomputation and Physics of Complex Systems (BIFI), University of Zaragoza, ARAID Foundation, Zaragoza, Spain

${ }^{5}$ Copenhagen Center for Glycomics, Department of Cellular and Molecular Medicine, School of Dentistry, University of Copenhagen, Copenhagen, Denmark

${ }^{6}$ Laboratorio de Microscopías Avanzada (LMA), University of Zaragoza, Zaragoza, Spain

\section{ACKNOWLEDGEMENTS}

R.H-G thanks ARAID, MEC (CTQ2013-44367-C2-2-P and BFU2016-75633-P), Gobierno de Aragón (E34_R17 and LMP58_18) with FEDER (2014-2020) funds for 'Building Europe from Aragón' for financial support and the FP7 (2007-2013) under BioStruct-X (grant agreement No. 283570 and BIOSTRUCTX_5186). A.C-G is thankful to Consejería de Economía, Conocimiento, Empresas y Universidad of the Junta de Andalucía (Excellence Research Project P18-RT-1679) and the Oficina de Transferencia de Resultados de Investigación of the University of Granada (PR/17/006 project) for financial support. B.R-R gratefully acknowledges funding from the European Union's Horizon 2020 research and innovation programme under Marie Sklodowska-Curie Grant Agreement No. 754446 and UGR Research and Knowledge Transfer Fund - Athenea3i. The authors thank Dr Vikki Cantrill for her help proof-reading this manuscript. 


\section{Abstract}

Up-regulated choline metabolism, characterized by an increase in phosphocholine (PCho), is a hallmark of oncogenesis and tumor progression. Choline kinase (ChoK), the enzyme responsible for PCho synthesis, has consequently become of a promising drug target for cancer therapy and as such a significant number of ChoK inhibitors have been developed over the last few decades. More recently, due to the role of this enzyme in other pathologies, ChoK inhibitors have also been used in new therapeutic approaches against malaria and rheumatoid arthritis. Here, we review research results in the field of ChoK $\alpha$ inhibitors from their synthesis to the molecular basis of their binding mode. Strategies for the development of inhibitors and their selectivity on ChoK $\alpha$ over ChoK $\beta$, the plasticity of the choline-binding site, the discovery of new exploitable binding sites, and the allosteric properties of this enzyme are highlighted. The outcomes summarized in this review will be a useful guide to develop new multi-functional potent drugs for the treatment of various human diseases.

Keywords: Choline kinase, inhibitors, structure-based drug design, cancer, malaria

\section{INTRODUCTION}

Cancer is a health and economic burden, and a leading cause of death worldwide that accounted for an estimated 9.6 million deaths in $2018 .{ }^{1}$ Despite of the vast complexity of biochemical mechanisms underlying this group of diseases, magnetic resonance spectroscopic (NMR) studies have revealed a common characteristic in some breast, lung, colon, bladder, prostate and ovarian cancers that can be used in the design of new antitumor drugs: an increase of phosphocholine (PCho) and total choline (Cho)-containing compounds relative to healthy tissues. ${ }^{2-6}$ This phenomenon is so pronounced that $\left[{ }^{11} \mathrm{C}\right]$-Cho and $\left[{ }^{18} \mathrm{~F}\right]-$ Cho are used in Positron Emission Tomography (PET) as tracers to diagnose, monitor and prognose solid tumors. ${ }^{7-11}$

PCho is a precursor of phosphatidylcholine (PtdCho), which is a major component of mammalian and some prokaryotic cell membranes and also the precursor of signaling molecules, such as phosphatidic acid that triggers the mitogenic signal. ${ }^{12-15} \mathrm{PCho}$ is synthesized by choline kinase (ChoK), a cytoplasmic 
enzyme, the expression of which is regulated by the concentration of intracellular Cho, ${ }^{16}$ hypoxiainducible factor-1 $\alpha$ (HIF1- $\alpha),{ }^{17}$ hormones, ${ }^{18}$ cytokines and growth factors, such as EGF, ${ }^{19}$ insulin ${ }^{19}$ and prolactin. ${ }^{20}$ Human ChoK (hChoK) exists in nature as three isoforms ( $\alpha 1, \alpha 2$ and $\beta$ ) that generate active homo or heterodimers, which catalyze the conversion of Cho into PCho by using ATP and $\mathrm{Mg}^{2+}$ as a cofactor. The catalytic mechanism has yet to be determined, but there are currently two proposed mechanisms. The first mechanism requires the formation of a ternary complex, whereas the second involves the formation of a phosphorylated enzyme intermediate (see section 4.3 for a detailed analysis of the two potential catalytic mechanisms). ${ }^{21,22}$

hChoK $\alpha 1$ and hChoKa2, encompassing 457 and 439 amino acids, respectively, are the product of chk$\alpha$ gene alternative splicing, whereas hChoK $\beta$ (395 amino acids in length) is encoded by chk- $\beta$. Despite the high grade of identity (56\%) at the amino acid level between hChoK $\alpha$ and $h C h o K \beta$, both enzymes have proven differences in the lipid metabolism and their role in carcinogenesis. ${ }^{23,24}$ hChoK $\alpha$ is overexpressed in some of the most common cancers and has oncogenic activity when overexpressed in human cells, yet most studies claim that $\mathrm{hChoK} \beta$ does not contribute to oncogenic transformation. ${ }^{25-}$ 30

As a result of the relevance of hChoKa in human carcinogenesis, many hChoK $\alpha$ inhibitors have been synthesized by means of different chemical approaches. The inhibition of hChoK $\alpha$ leads to an indirect decrease of PtdCho and in turn, to cell proliferation arrest through apoptosis, which is an efficient antitumor strategy. ${ }^{31,32}$

The first series of hChoK inhibitors were designed based on the results from quantitative structureactivity relationship (QSAR) models of Cho uptake inhibitor hemicholinium-3 (HC-3; Figure 1). ${ }^{31}$ HC-3 was modest in terms of activity ( $\left(\mathrm{C}_{50} \text { and } \mathrm{EC}_{50} \text { values of } 57 \text { and } 500 \mu \mathrm{M} \text {, respectively) }\right)^{33}$ and neuro- and lung-toxic at therapeutic doses. HC-3 consists of a biphenyl spacer and two oxazonium cationic rings $^{34,35}$ that were subsequently pharmacomodulated to generate more active and safer derivatives in vitro and in vivo. MN58b (Figure 1), a derivative of HC-3, was identified as a lead molecule with potent antiproliferative activity in vitro and efficient antitumoral activity in human colon cancer 
xenografts in nude mice..$^{31,33,36}$ In addition, MN58b allowed a deeper understanding of the mechanism of action of this novel class of antitumor drugs and served as a template for the preparation of new compounds. In a later stage, the replacement of pyridinium by quinolinium as cationic moieties led to RSM-932A ${ }^{37}$ (aka TCD-717; Figure 1) that provided the best results in vitro and in vivo. ${ }^{38}$ In fact, RSM932A is the first hChoKa inhibitor reaching Phase I clinical trials for advanced solid tumor treatment (see NCT01215864 for further information). ${ }^{39}$

The elucidation of the hChoK $\alpha 2$ crystal structure ${ }^{21}$ in 2006 and the discovery of the hChoKa1 crystal structure complexed with HC- $3^{40}$ in 2010 opened the possibility of structure-based drug design of new compounds. In recent years, the elucidation of the crystal structures of some of these inhibitors bound to hChoK $\alpha 1$ have been used for the development of completely different new scaffolds based on computational methodologies, such as docking, virtual screening, high-throughput screening and fragment-based drug design.

The similarities between the active sites of ChoK orthologs have demonstrated the possibility of using ChoK $\alpha$ inhibitors to block the growth of some parasite species, such as Plasmodium falciparum. ${ }^{41-43}$ These facts together with the recently reported high expression of hChoK $\alpha$ in the synovium of patients with rheumatoid arthritis and in fibroblast-like synoviocytes ${ }^{44}$ reveal the utility of ChoK as a therapeutic target to treat other prevalent diseases, such as malaria and rheumatoid arthritis.

Due to the increasing information on ChoK as a druggable anticancer, antimalarial and antirheumatic target, several reviews have been published. ${ }^{3,4,11,28,32}$ However, most of these reviews focus on the biological role of the enzyme and its importance as a diagnostic/prognostic tracer. Herein, we review recent advances in the discovery of ChoK inhibitors by applying different methodologies, such as HC-3 molecular modulation, screening of natural products or computer-based drug design as well as the structural aspects of inhibitor recognition by hChoK $\alpha$ and its impact in the structure-guided drug design for the treatment of different diseases. 


\section{INHIBITORS BASED ON HC-3 STRUCTURE MODIFICATIONS: THE USE OF MOLECULAR MODULATION}

\section{IN DRUG DESIGN}

As already mentioned, the synthesis of several derivatives was first based on structural modifications of HC-3 that led to biscationic, triscationic and macrocyclic compounds, such as cyclophanes and bicyclophanes (Figure 1). ${ }^{45-53}$ All these molecules are symmetrical with pyridinium or quinolinium moieties connected through an aromatic linker. As demonstrated by preclinical candidate TCD-717, this approach is successful and justifies its use in the design of new hChoK inhibitors.

\subsection{Symmetric bispyridinium, bisquinolinium and bisquinuclidinium derivatives}

Based on QSAR studies that demonstrated that the presence of an electron-releasing group at position 4 of the pyridinium ring favors both $\mathrm{hChoK}$ inhibitory and antiproliferative activities, ${ }^{54} \mathrm{Gomez-Perez} \mathrm{et}$ al. prepared a new series of ten bispyridinium and bisquinolinium derivatives that all contained cyclic or acyclic amino groups at position 4 of the heterocycle. ${ }^{55}$ All structures showed a potent inhibitory activity against $\mathrm{hChoK} \alpha 2$ with $\mathrm{IC}_{50}$ values in the range $0.08-1.35 \mu \mathrm{M}$. Compound 1 (Figure 2 ) was identified as the most potent hChoK inhibitor of this series with $\mathrm{IC}_{50}$ value of $80 \mathrm{nM}$, a ten-fold greater potency than $\mathrm{HC}-3\left(\mathrm{IC}_{50}=0.92 \mu \mathrm{M}\right)$ under the same assay conditions. Kinetic enzymatic assays indicated a mixed and predominantly competitive mechanism of inhibition for this compound, which exhibited a remarkable antiproliferative activity $\left(E C_{50}=1.5 \mu \mathrm{M}\right)$ against the human breast cancer SKBR3 cell line. Following the classical bioisosteric equivalence between bipyridine and biphenyl, which has been identified as one of the most suitable spacers for development of symmetric ChoK inhibitors, Castro et al. prepared a new series of biscationic hChoK inhibitors (Figure 2). ${ }^{56}$ The introduction of two nitrogen atoms within the linker would improve the polarity of the structures and the affinity for the enzyme owing to the likely formation of additional hydrogen bonds with hChoKa1 active site residues, as predicted by computational studies. Regarding the inhibitory activity assayed on hChoKa1 enzyme, quinolinium and pyridinium derivatives presented a good inhibitory profile but no activity was observed for quinuclidinium structures. In turn, the potency of these derivatives was clearly boosted with the presence of an $\mathrm{N}$-methylaniline fragment at position 4 of the pyridinium or quinolinium ring 
with $I C_{50}$ values ranging 3.9-9.0 $\mu \mathrm{M}$. Seven cancer-cell lines of different origin, namely cervix, leukemia, breast, lung and colon, were used to test the antiproliferative activity of the structures, proving to be concordant with hChoKa1 inhibition. Although quinuclidinium and pyridinium derivatives had no or little effect respectively, quinolinium derivatives were in general potent antiproliferative compounds, especially against leukemia and colon cancer cells. As expected by enzyme inhibitory activities, the presence of an $\mathrm{N}$-methylaniline fragment at position 4 of the quinolinium ring led to the greatest activities $\left(E C_{50} S=0.28-16.7 \mu \mathrm{M}\right)$ likely related to the combination of the direct effect of this group in the inhibition together with high lipophilicity conferred by it (clogPs $=2.70-3.45)$. Most active compound 2 (Figure 2) showed comparable activity to RSM-932A against human promyelocyticleukemia (HL-60) and colon carcinoma (HT-29) cell lines despite presenting less inhibitory capacity under the same experimental conditions (IC $\mathrm{C}_{50} \mathrm{~S}=9.02$ versus $\left.0.78 \mu \mathrm{M}\right)$. Importantly, compound $\mathbf{2}$ had low toxicity in non-transformed cells as previously observed for MN58b or RSM932A. Flow cytometry and immunoblot analysis with cervix and leukemia cancer cells confirmed that 2 induces caspase-3-dependent apoptosis with a marked mitochondrial membrane depolarization.

Considering previous evidence that supports bioisosterism as an effective tool to improve the binding to hChoK, Schiaffino-Ortega et al. reported a series of symmetric biscationic compounds with an oxygen-containing linker (1,2-diphenoxyethane) in an attempt to obtain more potent and soluble structures. ${ }^{57}$ Among the different cationic moieties included in this work, quinuclidinium-derived structures proved to be weak hChoKa1 inhibitors in agreement with previous data. ${ }^{56}$ However, pyridinium and quinolinium derivatives showed a moderate hChoK $\alpha 1$ inhibition activity favored by the presence of an alkylamine or a cycloalkylamine at the position 4 of both cationic moieties. Compounds 3a and $\mathbf{3 b}$ (Figure 2) that present 4-(dimethylamino)pyridinium and 4-(pyrrolidin-1-yl)quinolinium, respectively, are the most potent $\mathrm{hChoK}$ inhibitors of this series with comparable activities to reference structures MN58b and RSM-932A ( $\mathrm{IC}_{50} \mathrm{~S}=1.00$ and $0.92 \mu \mathrm{M}$ versus 0.78 and $1.92 \mu \mathrm{M}$, respectively). By means of tryptophan fluorescence quenching, $K_{\mathrm{d}}$ values of both compounds were calculated, rendering a high affinity for hChoKa1 enzyme ( $K_{d} S=0.70$ and $0.35 \mu \mathrm{M}$, for $\mathbf{3 a}$ and $\mathbf{3 b}$, respectively). Regarding the antiproliferative activity determined in a panel of 9 different cancer cell lines, pyridinium 
derivatives provided better results than quinolinium derivatives, with poor activity for quinuclidiniumderived structures. Aligned with hChoK inhibition data, 3a offered the best antiproliferative profile within the pyridinidium family with $\mathrm{EC}_{50}$ values in the nanomolar range $\left(\mathrm{EC}_{50} \mathrm{~S}=0.027-0.12 \mu \mathrm{M}\right)$. Compound 3a was also investigated in non-tumor cells proving low toxicity against human fibroblast and HUVEC cells. In relation to the mechanism of cell death, this molecule caused a marked G1 cellcycle arrest with concomitant reduction of the S phase in Jurkat, MCF-7 and MDA-MB-231 cancer cells. Surprisingly, treatment with compound $\mathbf{3 a}$ induced just a slight increment of apoptotic cells in Jurkat cells, with no increase in MCF-7 and MDA-MB-231 cells.

Based on the marked activity of 3a on cell proliferation, additional studies were performed in a panel of human T-cell acute lymphoblastic leukemia (T-ALL) cell lines and primary cultures of pediatric T-ALL patients in which the consistent overexpression of $\mathrm{hChoK \alpha}$ isoform was previously proved. ${ }^{58}$ Compound 3a exhibited remarkable antiproliferative activity in all the T-cell leukemia cell lines tested $\left(E_{50}\right.$ values $\left.0.9-479 \mathrm{nM}\right)$ and a good efficacy to inhibit cell growth in primary cell cultures ( $E C_{50}$ values 0.52-6.07 $\mu \mathrm{M})$. In agreement with previous results, 3a induced a block of the cell cycle in G1 with a consequent decrease of the $S$ phase that ultimately led to apoptotic cell death following the mitochondrial pathway. Reverse Phase Protein Array analysis was performed to evaluate the signaling pathways activated by $\mathbf{3 a}$ in T-ALL cell lines, which revealed rapid activating phosphorylation of AMPactivated protein kinase (AMPK) followed by deregulation of the mTOR pathway. Drug combination assays with dexamethasone and L-asparaginase, commonly used in T-ALL therapeutic protocols, proved that 3a enhances the chemotherapeutic effects of both drugs, especially L-asparaginase.

In a subsequent publication, the effect of $3 a$ was further investigated in vitro and in vivo in breast cancer, in which the overexpression and hyperactivation of hChoKa is associated with tumor progression, invasion, and metastasis. ${ }^{59}$ Among the main findings, it was demonstrated that $\mathbf{3 a}$ treatment significantly reduced PCho levels in MDA-MB-231 cells and strongly affected MDA-MB-231, MDA-MB-468 and MCF-7 cell proliferation with a G1-phase cell-cycle arrest. Additionally, 3a was able to suppress migration and invasion in the highly metastatic MDA-MB-231 cell line. Consistent with the 
previously obtained results in T-ALL cell lines, 3a activated the AMPK-mTOR signaling pathway and triggered cellular senescence in the 3 breast-cancer cell lines tested. In vivo, compound 3a caused a significant reduction of the tumor volume in a syngeneic orthotopic E0771 mouse model of breast cancer as well as the number of micro and macrometastases in the lungs of treated mice.

Later, due to the involvement of hChoK in the biosynthesis of PtdCho through Kennedy's pathway, Sola-Leyva et al. carried out a thorough study of the effect of $\mathbf{3} \mathbf{a}$ and $\mathbf{3} \mathbf{b}$ on the lipid metabolism in HepG2 cells. ${ }^{60}$ Results demonstrated that $\mathbf{3 a}$ and $\mathbf{3} \mathbf{b}$ clearly inhibited Cho uptake and incorporation of radiolabeled Cho into PtdCho. Both inhibitors caused a decrease in cholesterogenic activity and alterations in the expression of proteins related to cholesterol homeostasis with a more pronounced effect for $\mathbf{3} \mathbf{b}$. Treatment of HepG2 cells with $\mathbf{3} \mathbf{a}$ and $\mathbf{3} \mathbf{b}$ also resulted in a lowering of hChoKa protein levels and, in turn, PtdCho levels. The investigation of the AMPK signaling pathway confirmed that these inhibitors increased the phosphorylation and activation of AMPK in HepG2 cells.

By using 4-dimethylaminopyridinium as choline-mimicking moieties, Trousil et al. prepared a set of symmetric hChoK inhibitors that bears different alkyl linkers. ${ }^{61}$ The results of the inhibitory activity against recombinant hChoKa 2 proved that the potency of these structures is directly related to the length of the alkyl chain, with an 18-fold increase in the inhibitory capacity when the linker is elongated from 8 to 14 carbons. One of the competitive inhibitors included in this work, ICL-CCIC-0019 (Figure 2), was selected for rigorous pharmacological assessment due to its inhibitory potency $\left(\mathrm{IC}_{50}=0.27 \pm 0.06\right.$ $\mu \mathrm{M})$ and anticancer properties in $\mathrm{HCT} 116$ cells $\left(\mathrm{EC}_{50}=0.64 \pm 0.05 \mu \mathrm{M}\right)$. This small inhibitor selectively targets $\mathrm{hChoK} \alpha 2$ as shown in a multi-kinase screening and rendered a potent antiproliferative activity ( $\mathrm{EC}_{50}$ values from 0.0389 to $16.2 \mu \mathrm{M}$ ) across 60 cell lines included in the National Cancer Institute panel (NCI-60), with significant sensitivity against breast and non-small lung cancer cell lines..$^{62}$ ICL-CCIC-0019 reduced the uptake of $\left[{ }^{3} \mathrm{H}\right]$-Cho into $\mathrm{HCT} 116$ cells $\left(\mathrm{EC}_{50}=0.98 \pm 0.24 \mu \mathrm{M}\right)$, decreasing intracellular PCho levels and the incorporation of labelled Cho into lipids. Treatment with ICL-CCIC-0019 for 48 hours induced a marked G1 cell cycle arrest and caspase-3/7-mediated apoptosis in colon cancer HCT116 cells. Analysis of key regulators of endoplasmic reticulum (ER) stress showed that ICL-CCIC-0019 caused a profound ER stress response with no formation of reactive oxygen species. Studies of ICL-CCIC-0019 
in HCT116 xenograft bearing mice showed potent antitumor activity in vivo directly related to hChoK $\alpha$ inhibition as confirmed by PET by using the radiotracer $\left[{ }^{18} \mathrm{~F}\right]-\mathrm{D} 4-\mathrm{FCH}$ that targets the same enzyme. An in-depth study of the metabolic consequences of hChoK inhibition by ICL-CCIC-0019 revealed a previously unappreciated effect of Cho metabolism on mitochondria function. ${ }^{62}$ Drug treatment elicited a loss of mitochondria membrane potential and dose-related suppression of mitochondrial respiration, which is concomitant with increased glycolysis.

With a one-pot approach, Martín-Cantalejo et al. synthesized a series of bispyridinium salts of 4,4'bispyridyl-5,5'perfluoroalkyl-2,2-bisoxazoles from symmetric diamides.$^{63}$ These molecules, structurally related to HC-3 and its bispyridinium derivatives, were assayed as inhibitors of hChoK $\alpha$ and antiproliferative agents against human colon adenocarcinoma HT-29 cells, which resulted in an acceptable correlation between both biological activities. Regarding the cytotoxicity, the presence of a p-biphenyl linker and a $\mathrm{CF}_{3} \mathrm{CF}_{2}$ chain at position 5 of both oxazole rings clearly enhanced the antiproliferative activity of the structures. The best $\mathrm{EC}_{50}$ value was achieved with a $\mathrm{CH}_{2}-\mathrm{CH}_{2} \mathrm{OH}$ substituent at the pyridine nitrogen, which has the ability to form H-bonds (4a; Figure 2, HT-29 EC $50=$ $0.84 \pm 0.005)$. In terms of hChoK $\alpha$ inhibition, no correlation between the activity of the molecules and either the linker or perfluoroalkyl substituent was observed. However, the inhibition capacity was clearly improved with the use of $\mathrm{CH}_{2}-\mathrm{CON}\left(\mathrm{CH}_{3}\right)_{2}$ as alkylating agent for the quaternization of the pyridine (4b; Figure $2, I_{50}=0.30 \pm 0.003$ ).

\subsection{Symmetric bisindolium derivatives}

To image the hChoK expression and function in vivo, Arlauckas et al. designed a carbocyanine-derived hChoKa inhibitor, termed JAS239 (Figure 2), based on the existing structural similarities of previously reported bis-symmetric hChoK $\alpha$ inhibitors and carbocyanine dyes ${ }^{64}$ To this end, hydroxyethyl alkyl groups that mimic Cho structure were attached at the nitrogen atoms of both indolium rings present on a carbocyanine dye, the 7-carbon spacer of which confers fluorescence in the near infrared (NIR) range. 
In vitro experiments evidenced that JAS239 showed comparable potency to reference compound MN58b both inhibiting phosphorylation of ${ }^{14} \mathrm{C}$-labeled Cho and blocking cell growth in breast cancer cell lines. The hChoK inhibition was reversible by the addition of unlabeled Cho at 2 hours posttreatment, which demonstrates that JAS239 acts as a competitive inhibitor. This competitive mechanism was later corroborated by fluorescence imaging; cells pre-treated with a hChoKa-specific antibody presented a marked reduction in JAS239 retention. ${ }^{65}$ The analysis of the cellular uptake by means of fluorimetry and confocal microscopy demonstrated that JAS239 rapidly enters breast cancer cells independently of Cho transporters and is accumulated in the cytosolic space.

In vivo, the inherent optical properties of this small-molecule inhibitor allowed the study of its biodistribution in orthotopic 4175-Luc+ tumors xenografts. ${ }^{65}$ Intratumoral NIR fluorescence was detectable in JAS239-injected mice, although it was also noticeable in other major organs. Moreover, JAS239 accumulation was significantly higher in hChoKa-overexpressing breast tumors relative to empty vector counterparts. In a similar manner as found for MN58b, JAS239 significantly decreased total Cho-containing metabolite levels and tumor growth rate of orthotopically-implanted 4175-Luc+ tumors with no observable toxicity up to $4 \mathrm{mg} / \mathrm{kg}$. Histological assessment of the tumor status revealed marked cell density reduction and an increase of caspase-3 positive cells in response to JAS239.

\section{IN VITRO SCREENING OF NATURAL PRODUCTS}

Natural products and their derivatives have also been considered as potential hChoK inhibitors. Triterpenequinonemethides (TPQs) are bioactive compounds isolated from plants of the Celastraceae family that possess a pentacyclic D: A-friedo-nor-oleanane-type skeleton. Due to the presence of two hydrophilic and electron-deficient electronic regions located on the $A$ and $E$ rings and a hydrophobic zone located on the $B, C$ and $D$ rings, natural TPQs may fit the structural requirements for being hChoK $\alpha$ inhibitors. Accordingly, Estevez-Braun et al. prepared a set of semi-synthetic TPQ derivatives derived from natural ones by chemical modifications, such as bromination, oxime formation and

esterification. ${ }^{66}$ Both natural and semi-synthetic derivatives were assayed against partially purified 
recombinant hChoK $\alpha 1$ expressed in $\mathrm{E}$. coli, and 14 out of 59 compounds had $\mathrm{IC}_{50}$ values in the range 0.6-8.6 $\mu \mathrm{M}$. Preliminary structure-activity relationships led them to establish that compounds derived from naturally occurring $22 \beta$-hydroxy-tingenone (Figure 2 ) are more active than those derived from either tingenone or pristimerine. The most potent hChoK inhibitors $\left(\mathrm{IC}_{50} \mathrm{~S} \leq 10 \mu \mathrm{M}\right)$ were also analyzed as antiproliferative agents against the human colon adenocarcinoma HT-29 cell line. Among them, 22 $\beta$ hydroxy-tingenone showed an $\mathrm{EC}_{50}$ value of $6.5 \mu \mathrm{M}$ and was selected for further in vivo investigations. Toxicity studies performed in CD1 nude mice demonstrated a lethal dose $\left(L_{50}\right)$ of $12.5 \mathrm{mg} / \mathrm{kg}$. Remarkably, its intraperitoneal administration (at $7.5 \mathrm{mg} / \mathrm{kg}$ in a daily basis for 5 days) resulted in a 75 \% reduction of tumor growth in HT-29 human colon carcinoma xenograft.

\section{HARNESSING THE POTENTIAL OF CRYSTALLOGRAPHIC STUDIES FOR THE DESIGN OF NEW CHOK INHIBITORS}

The hChoK three-dimensional structures determined by X-ray crystallography (Figure 3 ) have enabled the visualization of the $\alpha$ and $\beta$ isoforms architecture, which has ultimately provided the understanding of its catalytic and allosteric mechanisms, and the molecular basis for the development of tailor-made inhibitors. Moreover, these structures have also inspired new hypotheses to probe the binding modes of plausible inhibitors in alternative pockets and the feasibility of cross-inhibition of the hChoK $\alpha$ inhibitors for the treatment of infectious diseases. Altogether, this section evidences the rapid growth on the structural information of ChoK in recent years and its impact in the structure-based design of potent inhibitors.

\subsection{ChoK X-ray crystal structures reveal ATP and Cho binding sites}

Knowledge of the tridimensional structure of a therapeutic target speeds up the development of more selective drugs. The crystal structure of Caenorhabditis elegans ChoK (CeChoK) apo-form (PDB ID: 1NW1) revealed for the first time the architecture of the active site.$^{67}$ Although the ATP pocket was solvent exposed, the Cho binding site was a deep hydrophobic groove located in the proximity of the ATP pocket. Based on the 3D structure of CeChoK, Milanese et al. prepared a homology model of 
hChoK $\alpha$ that was employed for docking studies of reported symmetric biscationic inhibitors. ${ }^{68}$ In this article, the authors suggest that the compounds bind simultaneously in both the ATP and Cho putative binding sites.

At the same time that the hChoK homology model was published, three crystal structures of hChoKa2 isoform were also reported: the apo-form (PDB ID: 2CKO) and two binary complexes that contain the products of the enzymatic reaction, ADP (PDB ID: 2CKP) and PCho (PDB ID: 2CKQ). ${ }^{21}$ These structures unambiguously identified both the ATP and Cho binding pockets, and the residues tethering these products.

ADP binds into a cleft between the $\mathrm{N}$ and $\mathrm{C}$-terminal lobes and is stabilized by a network of hydrogen bonds with R117, N122, R146, E206, Q207, 1209, S211, R213 and D330 (Figure 4). In addition, ADP is also tethered by L124, L144, L313, and F208 through hydrophobic and $\pi-\pi$ interactions. The Cho binding site is a deep hydrophobic groove formed by Y333, Y354, W420, W423 and Y440 (Figure 4) that comprises a rim of acidic residues (E215, E217, E218, E309, E349, E357, E434 and D353). PCho is stabilized by $\pi$-cation interactions between quaternary ammonium moiety and the aromatic residues of the hydrophobic cleft (Y333, Y354, W420, W423 and Y440), and hydrogen bonds between its phosphate group and residues L120, S121, Q308 and N311.

\subsection{Structural differences between the active sites of $h$ ChoK $\alpha 1$ and $h$ ChoK $\beta$}

The publication of the crystal structure of hChoK $\alpha 1$ and $\beta$ isoforms in complex with HC-3 and phosphoHC-3 (pHC-3), respectively (Figure 5; PDB IDs: 3 G15 and 3FEG), ${ }^{40}$ allowed a deeper understanding of the structural differences between both isoforms. Both structures revealed that HC-3 and pHC-3 bind at the Cho binding site, which provides a rationale of its inhibitory properties. However, $\mathrm{HC}-3$ is a better inhibitor of $h C h o K \alpha 1$ than $h C h o K \beta$ as observed from the $K_{d}$ values (450-fold lower for $h C h o K \alpha 1$ versus hChoK $\beta) .{ }^{40} \mathrm{~A}$ thorough inspection of both crystal structures determined that the selectivity of HC-3 on $h C h o K \alpha 1$ in detriment of $h C h o K \beta$ was explained by reduced flexibility of W353 in hChoK $\beta$ relative to its homologue W420 in hChoKa1. Although a more rigid amino acid, such as F352 is close to W353 and limited its motion, homologue residue L419 in hChoK $\alpha 1$ allows W420 to have more freedom of motion 
allowing the flexibility seen in W420 to interact optimally to HC-3. This reduction in flexibility of the W353 likely caused the different and non-optimal binding mode of the pHC-3 oxazinium ring and in turn explained the poor affinity of HC-3 towards hChoK $\beta .{ }^{40}$

\subsection{Proposed catalytic mechanisms based on structural and kinetic studies}

The understanding of the catalytic mechanism and the residues participating in binding and catalysis is of enormous importance for the design of new drugs.

To date, two catalytic mechanisms have been proposed for ChoK. The first mechanism was reported in 2006 based on a model from X-ray crystal structures of hChoKa2 in the apo form (PDB ID: 2CKO), complexed with ATP (PDB ID: 2CKP) and Cho (PDB ID: 2CKQ). ${ }^{21}$ This hypothesis was based on the formation of a tertiary complex of hChoK $\alpha$ with ATP, Cho and $\mathrm{Mg}^{2+}$ prior to phosphorylation. The authors, according to their mutagenesis data, proposed that D306 and D330 played an essential role in the reaction mechanism by coordinating the magnesium ions that bridge the ADP and PCho molecules (Figure 6). However, no information was provided to account for the binding order of the substrates, the phosphorylation step and product release.

Secondly and more recently, Hudson et al. supported a double displacement catalytic mechanism based on substrate kinetic studies (Figure 7). ${ }^{22}$ According to their hypothesis, the $\gamma$-phosphate of ATP is transferred to Cho in two steps through the formation of a phospho-enzyme intermediate. In the first step, ATP binds to ChoK and then D306 develops a magnesium-mediated nucleophilic attack to the $\gamma$-phosphate of ATP. Once the D306 is phosphorylated, ADP is released from the active site and Cho enters its binding pocket where the transfer of the phosphate group from the phospho-D306 (PD306) to Cho takes place. ChoK undergoes conformational change to release PCho. Such a mechanism offers great potential for the design of new compounds that bind into different enzyme intermediates according to the phosphorylated states of D306.

Even though the two catalytic mechanisms are different, both of them have been observed in the kinase superfamily and share the fundamental role of D306 in catalysis. ${ }^{69}$ 


\subsection{Structure-based rational drug design}

\subsubsection{Non-symmetric pyridinium derivatives}

Based on the information provided by the hChoK $\alpha 2$ isoform crystal structure, Rubio-Ruiz et al. prepared the first series of non-symmetric hChoK inhibitors. ${ }^{70,71}$ These rationally designed compounds contain a 4-substituted pyridinium system bonded to an adenine moiety through linkers of different lengths. The pyridinium moiety would act as a cationic head resembling the Cho structure, whereas the adenine moiety would mimic the ATP adenine fragment. The N9 and N3 adenine alkylated derivatives were isolated and their biological activity assessed. The inhibitory capacity was evaluated by using a radiometric assay that relies on the phosphorylation of [methyl $\left.-{ }^{14} \mathrm{C}\right] \mathrm{Cho}$ by hChoK enzyme, which was partly isolated from cytosol of human HepG2 cells. In general, the dimethylamine substituent at the position 4 of the pyridinium fragment led to higher hChoK inhibitory potency that was also favored by the presence of longer linkers, such as 1,4-diphenylbutane. On the contrary, the antiproliferative activity on HepG2 cells shown by 4-pyrrolidine derivatives was slightly greater than the activity reported by the dimethylamine analogs. Compounds $5 \mathbf{a}\left(I C_{50}=10.70 \pm 0.40 \mu \mathrm{M}\right)$ and $\mathbf{5 b}$ $\left(\mathrm{IC}_{50}=6.21 \pm 0.97 \mu \mathrm{M}\right)$ of the N9 and N3 series, respectively, were the most potent ChoK inhibitors (Figure 8). ${ }^{70}$ Docking studies performed in hChoKa1 (PDB ID: 3G15) suggested that 5 a bound simultaneously in both the ATP and Cho binding sites of hChoK $\alpha 1$ enzyme, which provides a rationale of its potency.

In a follow-up publication, Sahún-Roncero et al. reported the crystal structure of hChoKa1 in complex with 5a (PDB ID: 3ZM9), ${ }^{72}$ which confirmed the binding mode previously predicted by docking studies (Figure 8). A comparison of the overall structure of hChoK $\alpha 1-5$ a crystal structure with the apo structure revealed large conformational changes. ${ }^{72}$ In addition, the asymmetry of the two monomers that form the dimer of hChoKa1-5a provided the structural basis of the negative cooperativity observed by isothermal titration calorimetry. ${ }^{72}$ This experiment evidenced that compound 5 a firstly bound to one of the two monomers of hChoK $\alpha 1$ with a dissociation constant $\mathrm{K}_{\mathrm{d} 1}$ of $38 \mathrm{nM}$, whereas the binding to the second monomer led to a higher $\mathrm{K}_{\mathrm{d} 2}$ of $190 \mathrm{nM}$. Besides these evidences, molecular dynamics (MD) 
and anisotropic elastic network studies on the hChoKa1-5a crystal structure confirmed the negativecooperativity allosterism. ${ }^{72} \mathrm{MD}$ simulations revealed that the two monomers, alternatively assumed an open/semi-open or closed state with a fast interconversion rate. Taken together, these data explained the molecular mechanism of the negative cooperativity phenomenon, the inter-monomer coupling, and set the foundation to design better and more selective inhibitors.

Through a chemical deconvolution approach based on hChoK $\alpha 1-5 a$ and hChoK $\alpha 1-5 b$ crystal structures, it was shown that the adenine and 1-benzyl-4-(dimethylamino)pyridinium components were the most efficient fragments in binding to hChoK $\alpha 1$ (Figure 8$){ }^{73}$

This first library of non-symmetric hChoK inhibitors evidenced a modest antiproliferative activity that was associated to their lack of lipophilicity. The introduction of a benzylthio substituent at position 6 of the purine heterocyclic ring led to a new series of hChoK inhibitors with an enhanced antiproliferative profile against human cervical carcinoma HeLa cell line. ${ }^{74}$ The cLogP values of these structures are at least 2-fold higher than the corresponding clogP values of the adenine series, which likely explains the higher antiproliferative effect observed. Treatment of HeLa cells with these compounds caused clear disturbances of cell cycle progression and induction of apoptosis in a caspase3-dependent process. ${ }^{74}$ As already mentioned, the presence of a dimethylamino group at the position 4 of the pyridinium ring led to a greater effect. However, the activity was also favored by short linkers, contrary to that previously observed for adenine counterparts. In fact, compound 6 (Figure 9), which possesses a benzene moiety as a spacer, is the most potent inhibitor in the series against ChoK $\alpha 1\left(\mathrm{IC}_{50}=0.4 \mu \mathrm{M} \pm 0.03\right) .{ }^{75}$

A similar approach was pursued by Trousil et al. that used alkyl linkers (C8 and C12) to bridge both the Cho and ATP-mimicking groups. ${ }^{61}$ Benzimidazole, adenine and purine were explored while keeping 4(dimethylamino) as the cationic moiety. The presence of an adenine (7, Figure 9) was associated with a significant increase in inhibitory activity $\left(\mathrm{IC}_{50}=0.8 \mu \mathrm{M}\right)$, although little correlation was found between the potency of hChoKa2 inhibition and the antiproliferative effect. 
Non-heterocyclic fragments were also considered as ATP mimetics in an attempt to obtain hChoK inhibitors with improved potency. Schiaffino-Ortega et al. ${ }^{76}$ prepared analogs to mimic the first series reported by Rubio et al. ${ }^{70}$ that contain a 3-aminophenol moiety instead of adenine. These analogs were chosen following preliminary computational studies that suggested that this moiety was inserted into the ATP binding site and was efficiently stabilized by hydrogen bonds with R146, D206, Q207 or 1209. Additionally, these studies suggested that either O- or N-linkages to 3-aminophenol would facilitate the simultaneous binding of all the designed derivatives into the Cho and ATP binding sites, excluding those structures with short linkers (i.e. benzene). Surprisingly, the biological results showed that molecules with long linkers are inactive or give rise to only a slight hChoK inhibitory effect, which invalidates the initial hypothesis. The authors rationalized that these results may be a consequence of the higher affinity of adenine fragment for the ATP binding site in relation to the 3-aminophenol affinity, which makes these inhibitors incapable of competing with ATP and occupy both binding sites. Compounds $8 \mathbf{a}$ and $\mathbf{8 b}$ (Figure 9) with $\mathrm{IC}_{50}$ S of $6.37 \pm 0.53$ and $7.89 \pm 0.05 \mu \mathrm{M}$, respectively, were the most active hChoK inhibitors of this series, which indicated that the 4-(dimethylamino)pyridinium cationic moiety and a biphenyl linker favored hChoK inhibition activity. ${ }^{76}$

\subsubsection{Non-Symmetric bispyridinium derivatives}

In line with the trend of preparing hChoK inhibitors with non-symmetric structure, Rubio-Ruiz et al. reported the first library of asymmetric bispyridinium derivatives to explore potential new binding modes to the enzyme. ${ }^{77}$ In these structures, 4-(4-chloro- $N$-methylanilino)-pyridinium, 1-benzyl-4(dimethylamino)pyridinium and 4-pyrrolidinopyridinium fragments were combined and both positive charged moieties were connected with variable linkers (Figure 10). All the molecules were similar in terms of hChoK inhibition, with $\mathrm{IC}_{50}$ values less than $2.5 \mu \mathrm{M}$ determined by measurement of the rates of incorporation of ${ }^{14} \mathrm{C}$ from [methyl- $\left.{ }^{14} \mathrm{C}\right] \mathrm{Cho}$ into PCho. The effects of these molecules on human cervical carcinoma HeLa cells proved that they efficiently inhibited cancer cell proliferation $\left(\mathrm{EC}_{50}<10\right.$ $\mu \mathrm{M})$ which resulted in disturbances in cell-cycle progression and induction of apoptosis. Particularly, 
treatment with 9a caused significant cell-cycle arrest in the G0/G1 phase and a $40 \%$ increase of the number of apoptotic cells in a caspase-3-dependent manner.

The crystal structures of hChoK $\alpha 1$ in complex with compounds 9a-9c (PDB ID: 4CG8, 4CGA and 4CG9, respectively) demonstrated that they bind exclusively to the Cho binding site. Particularly, the hChoK $\alpha 1$ X-ray crystal structure in complex with 9 a (Figure 10) enabled the discovery of a new binding pocket adjacent to the Cho binding site. ${ }^{77}$ This is a key indicator of the plasticity of the active site and can be exploited in the design of new selective and potent inhibitors. Compound 9a contains 4-(4chloro- $N$-methylanilino)pyridinium and 4-(dimethylamino)pyridinium fragments that are covalently bound through a 1,4-biphenylbutane linker. Due to its long flexible linker, this compound adopted a twisted conformation in the Cho binding site in which the 4-chloro- $N$-methylaniline moiety accessed an alternative hydrophobic pocket located at the back of the Cho binding site, which is formed by residues W248, T252, Y256, Y333, L419, W420, and W423 (Figure 10). This adjacent binding pocket was accessible prior induction of local conformational changes of several amino acids (Y256, E332, Y333, and W420). The rotation of the Y333 $\left(\approx 20^{\circ}\right), W 420\left(\approx 45-62^{\circ}\right)$ and Y256 $\left(\approx 90^{\circ}\right)$ side chains with respect to the position of the same residues in previous crystal structures was critical for inducing the aperture of the adjacent binding pocket. Access of the 4-chloro- $N$-methylaniline moiety to this new binding site together with the presence of a longer linker led to a better dissociation constant $\left(\mathrm{K}_{\mathrm{d}}\right)$ for 9a relative to the $K_{d} S$ of $9 \mathrm{~b}$ and $9 \mathrm{c}\left(0.11 \pm 0.01 \mu \mathrm{M}\right.$ versus $0.40 \pm 0.10$ and $0.62 \pm 0.15$, respectively) ${ }^{77}$ Overall, the authors concluded that flexible and long linkers in hChok $\alpha 1$ inhibitors increased the probability of accessing the adjacent binding pocket, which in turn improved selectivity and ligand affinity to give better in vitro biological effects.

\subsection{Computer-based drug design}

\subsubsection{Pharmacophore-based virtual screening}

Pharmacophore identification by a target-based approach is a potent tool for the discovery of new active compounds against a particular target. This strategy has been used for the discovery of new 
hChoKa1 binders based on the chemical structure of previous HC-3-derived inhibitors and the X-ray crystal structure of hChoK $\alpha 1$ in complex with HC-3 (PDB ID: 3G15). ${ }^{78}$

The identified pharmacophore model is formed by five moieties (three aromatic rings, a nitrogen atom and a positive charge; Figure 11) that are included in the 1-benzyl-4-(N-methylaniline)pyridinium fragment. This allowed the dissection of common structural features that were important for the binding mechanism and biological activity of the previous HC-3 derivatives (Figure 11). By using this pharmacophore model as a guide to search different virtual screenings, such as Enamine, Chembridge and Life Chemicals libraries, several molecules with low $\mu \mathrm{M}$ range binding affinities against hChoK $\alpha 1$ were discovered (Figure 11). Compound 10 was the most active $\left(K_{d}\right.$ of $\left.0.438 \pm 0.1 \mu \mathrm{M}\right)$, followed by 11 $\left(K_{d}=0.526 \pm 0.1 \mu \mathrm{M}\right)$ and $12\left(K_{d}=2.60 \pm 0.70 \mu \mathrm{M}\right)$. Protein crystallization studies demonstrated that 12 (PDB ID: 5AFV) binds to both the Cho and ATP binding sites, which suggests that this particular molecule has a dual mechanism in which it competes with both ATP/Mg ${ }^{+2}$ and Cho. ${ }^{78}$ According to the $K_{d}$ values, this pharmacophore model is efficient at discovering new hChoKa1 binders that can lead to a new generation of simpler and potent inhibitors by optimization of their chemical properties.

\subsubsection{In silico virtual screening of chemical libraries}

To identify potential hChoKa-interacting compounds, Clem et al. conducted an in silico screening of the ZINC Library by using the X-ray structure of hChoK $\alpha 2 .{ }^{79}$ The 16 best-score compounds in terms of binding with hChoK $\alpha$ were then tested in vitro. Only CK37 (Figure 12) significantly inhibited hChoK $\alpha$ activity in HeLa cell lysates and was selected for further experiments. This competitive inhibitor caused

a dose-dependent suppression of bacterially expressed recombinant hChoKa activity, which is completely reversed in the presence of increasing concentrations of Cho. Treatment of HeLa cells with CK37 at $10 \mu \mathrm{M}$ demonstrated the capacity of this molecule to suppress hChoK $\alpha$ activity in whole cells, which caused a reduction in Cho uptake and PCho production that in turn resulted in a decrease in the steady-state concentration of downstream Cho metabolites, such as phosphatidic acid. Additionally, it was found that exposure to $10 \mu \mathrm{M}$ of CK37 disrupted downstream MAPK and PI3K/AKT signaling by 
decreasing ERK and AKT-activating phosphorylations, altered the actin cytoskeletal organization and markedly attenuated plasma membrane ruffling. This inhibitor also suppressed cell growth in six neoplasic cell lines $\left(\mathrm{EC}_{50} \mathrm{~S}=5-10 \mu \mathrm{M}\right)$. This cytostatic activity was clearly dependent on the level of hChoKa expression because transfection of HeLa cells with a plasmid encoding hChoKa conferred resistance to the effects of CK37. Intraperitoneal administration of CK37 (0.08 mg/g per day for eight days in mice) significantly decreased tumor growth (48\% versus vehicle controls) and tumor PCho levels ( $51 \%$ versus vehicle controls) in a lung tumor xenograft mouse model.

\subsection{Fragment-based drug design}

Zech et al. successfully applied a fragment-based approach targeting hChoKa1 to generate new inhibitors. ${ }^{80}$ Saturation-transfer difference nuclear magnetic resonance spectroscopy was used as the primary screening technique to select hits that were further validated by biophysical methods like surface plasmon resonance (SPR) and docking and crystallography studies, which rendered lead-like compounds with nanomolar binding affinities and modest cellular potency. The most potent compound, 13 (Figure 13), inhibited hChoKa1 with an IC $5_{50}$ of $0.07 \mu \mathrm{M}$ and a $\mathrm{K}_{\mathrm{d}}$ of $0.01 \mu \mathrm{M}$. Structurally related compounds 14 and 15 (Figure $13, I C_{50}=0.09$ and $0.46 \mu \mathrm{M}$, respectively), proved growth inhibition and apoptosis induction on breast MDA-MB-468 and MDA-MB-415 cancer cell lines $\left(\mathrm{EC}_{50} \mathrm{~S}=\right.$ 2-9 $\mu \mathrm{M}$ ) with slight activity against non-transformed MCF-12A and MCF-10A cells ( $\left.E C_{50} \mathrm{~S}=18-40 \mu \mathrm{M}\right)$. Treatment with both small inhibitors for 24 hours caused a clear decrease in intracellular PCho levels in MDA-MB-468 and MDA-MB-415 cell lines, which confirmed the inhibition of hChoKa1.

One interesting chemical aspect of these new inhibitors is the absence of a non-permanent positive charge in the amino group, which is a major difference with most of the hChoKa1 inhibitors that had been previously synthesized. Although the positive charge in the compounds is important to achieve an efficient inhibition of the enzyme due to its resemblance to Cho, quaternary ammonium ions are also responsible for unspecific inhibition to other Cho-binding enzymes, which led to undesirable side effects. Most of the compounds identified as $\mathrm{hChoK} \alpha 1$ inhibitors from this fragment-based approach include basic amines (diazepanes or piperazines) that may be protonated depending on the local $\mathrm{pH}$. 
The crystal structure of hChoK $\alpha 1$ in complex with 13 (Figure 13; PDB ID: 5EQY) proved that the methyldiazepane fragment is a new pharmacophore that binds to the Cho binding site.$^{80}$ Interestingly, the methyldiazepane fragment establishes a hydrogen bond with D306, which has been suggested as the hChoKa1 catalytic base.

Although ATP binding site inhibitors were also identified through NMR fragment screening, their potency in comparison with the identified Cho-binding site inhibitors was much lower. This finding is in agreement with previous attempts to develop ATP mimics ${ }^{70,76}$ that confirmed the low druggability of the hChoK $\alpha 1$ ATP binding site.

\subsection{High-throughput screening of compound collection}

Inhibitor V-11-023907 (Figure 14) was discovered after applying high-throughput screening techniques with the Vertex compound collection. ${ }^{22}$ By means of a spectrophotometric assay, the $\mathrm{IC}_{50}$ value against hChoK $\alpha$ was determined as $0.47 \pm 0.08 \mu \mathrm{M}$. The hChoK $\alpha-\mathrm{V}$-11-023907 crystal structure (Figure 14, PDB ID: 4DA5) pinpointed the location of the compound in the Cho binding site and determined that the compound disrupted the role of the key catalytic D306 residue in catalysis by interaction through a hydrogen bond. Contrary to the crystal structure, kinetic studies indicated that compound V-11023907 was a non-competitive inhibitor of hChoKa with respect to Cho. These opposing conclusions have been rationalized by a second catalytic mechanism (see section 4.3 ) in which a double displacement is proposed. Accordingly to this mechanism, Cho binds to the phosphorylated form of the enzyme, whereas V-11-023907 may bind to the free enzyme. The existence of different enzyme intermediates based on the diverse protonation states of D306 offer the potential for compounds to potentially bind to different enzyme states, which could lead to the design of more potent and selective hChoKa inhibitors.

Then, a lead optimization approach performed by the same company (Vertex Pharmaceuticals) gave rise to compound V-11-0711 that inhibited recombinant hChoKa with an $\mathrm{IC}_{50}$ of $20 \mathrm{nM}$ and exhibited excellent selectivity against a panel of 50 kinases. ${ }^{81}$ Although V-11-0711 treatment led to substantial depletion of PCho levels in HeLa cells $\left(\mathrm{IC}_{50}<1 \mu \mathrm{M}\right)$, low levels of cell death were observed after 
incubation for $72 \mathrm{~h}$ at $10 \mu \mathrm{M}$. A detailed analysis of the cell cycle progression confirmed that this small molecule caused a slowdown in cell growth and division with great G1-phase accumulation. According to this data, the authors speculated that a non-catalytic protein scaffolding function of hChoKa would drive its oncogenic properties and suggested an antitumor strategy aimed at destabilizing the protein.

\subsection{The crystal structure of TCD-717 in complex with hChoKa opens the possibility of interrupting} protein-protein interactions in cancer

Despite the variety of hChoKa inhibitors that have been synthesized to date, only symmetric bisquinolinium compound TCD-717 has completed phase I clinical trials. ${ }^{38}$ In recent work, the inhibitor's interaction with hChoKa1 enzyme (PDB ID: 5W60) has been examined by X-ray crystallography and kinetic assays. ${ }^{82}$ Strikingly, TCD-717 bound near the surface of the enzyme (Figure 15) in a new binding site that was not exploited by the previously characterized hChoK $\alpha$ inhibitors. This fact together with other factors, such as its cell permeability, target specificity, in vivo bioavailability and low toxicity, could explain the significant in vivo effects of this compound, which inhibited hChoK $\alpha$ with an $\mathrm{IC}_{50}$ of $520 \pm 150 \mathrm{nM}$.

As described by Kall et al., TCD-717 did not bind to the Cho or ATP binding sites, and occupied a location near the surface. ${ }^{82}$ Residues Y148, A176, M177, E180, F200, P201, W248, T252, Y256, E332, Y333, and L419 accommodate TCD-717 through hydrophobic interactions (Figure 15), which precludes Cho binding due to the secondary structure movements induced. For instance, W248 and Y333 side chains experienced rotations $\left(\approx 100\right.$ and $\approx 35^{\circ}$, respectively, relative to the apo structure) to accommodate one of the TCD-717 quinolone rings. The other quinolone moiety of TCD-717 is stabilized by Y148 and M177 that also suffer conformational changes. The biphenyl linker helps to position the head groups of the compound in place and also displaces the side chains of E180 and E332, which avoids $\mathrm{Mg}^{2+}$ coordination and therefore ATP binding.

The phosphorylation of $\mathrm{Y} 333$ in a cSrc-dependent manner is linked to cancer progression. Phosphorylation may increase the enzymatic activity of $h C h o K \alpha$, which is a signal in the oncogenic pathway. Kall et al. have also very recently demonstrated, through SPR and crystallographic analysis, 
that ChoK $\alpha$ interacts with cSrc by a non-catalytic poly-proline motif (residues 60-69; see PDB ID: 6C4S) located in hChoK $\alpha \mathrm{N}$-terminal domain. ${ }^{83}$ This finding could lead to the design of new inhibitors that disrupt this hChoKa-cSrc interaction.

\subsection{Finding exploitable differences at the ChoK binding site between the human and pathogenic} species for drug design

The identification of high structural similarities in the active sites of ChoK orthologs led to the hypothesis that the hChoK $\alpha 1$ inhibitors could be repurposed for the treatment of infectious diseases in which ChoK might play an essential role.

With the aim of identifying similarities among the active sites of ChoKs from different species, the crystal structures of hChoKa1, Plasmodium falciparum ChoK (PfChoK) and Cryptosporidium parvum ChoK (CpChoK) were computationally analysed. ${ }^{84}$ Through sequence identity search and MD simulations, it was noted that the ATP and the Cho binding sites of hChoK $\alpha 1$, PfChoK and CpChoK were conserved (Figure 16), which suggests that previous compounds that inhibit the human enzyme may also interact with ChoK from different pathogens. This observation was substantiated with experimental in vitro fluorescence polarization assays that showed $\mathbf{1 6}$ and $\mathbf{1 7}$ presented similar $K_{d} \mathrm{~S}$ for hChoKa1, PfChoK and CpChoK (Figure 16).

A subsequent study by Serrán-Aguilera et $a l .{ }^{43}$ used previously reported non-symmetric bispyridinium derivatives $9 \mathrm{~d}$ and $\mathbf{9} \mathrm{e}^{77}$ (Figure 10 ) to probe whether these hChoK $\alpha 1$ inhibitors could target efficiently PfChoK and also propose a new mechanism of action of inhibition of ethanolamine kinase activity of $P f C h o K$, which led to a severe decrease in phosphatidylethanolamine levels within $P$. falciparum. ${ }^{43}$ The much lower dose of these compounds employed to inhibit $P$. falciparum growth suggested that these compounds could be exploited in the design of new clinical antimalarial drugs.

Finally and following the same rationale illustrated above, Zimmerman et $a l .{ }^{85}$ demonstrated that the active site of Streptococcus pneumoniae ChoK (SpChoK) was very similar to hChoK $\alpha 1$ and in turn inhibitors such as MN58b and RSM-932A acted as modest and potent inhibitors, respectively, at enzymatic and cellular level ( $\mathrm{IC}_{50 \mathrm{~S}}=197$ and $10 \mu \mathrm{M}$ and $\mathrm{EC}_{50} \mathrm{~S}=0.5$ and $0.4 \mu \mathrm{M}$, respectively). 
Altogether, all these results exemplified the potential benefit of repurposing $h C h o K \alpha 1$ inhibitors to tackle a wide range of infectious diseases.

\section{CONCLUSIONS AND FUTURE PERSPECTIVES}

The discovery of HC-3 as a lead compound and the later structural resolution of several hChoKs can be considered as the two major breakthroughs in the field of hChoKa inhibitors. The first one provided the starting point to design potent inhibitors, and the second one allowed a tailor-made design of specific inhibitors that exploited our knowledge of the active site architecture of this enzyme.

The development of most hChoK $\alpha$ inhibitors synthesized during the last 15 years has exploited information amassed from hChoK X-ray crystal structures. This knowledge, together with computational techniques, such as docking, virtual screening, high-throughput screening or molecular dynamics, have been key in the successful design of highly potent and specific hChoKa inhibitors that are considered lead compounds.

By applying all these medicinal chemistry, biophysical and computational approaches, we have learned a plethora of information on the biophysical characteristics of these enzymes and the key parts of the compounds to achieve potent inhibition: (a) HC-3 and subsequent compounds allowed us determine that potent inhibitors might contain a 1-benzyl-4-( $N$-methylaniline)pyridinium fragment that had a positive charge. However, the positive charge of the nitrogen atom of the inhibitors is not a requirement for inhibition as exemplified by 13; (b) the high selectivity of the inhibitors towards $h C h o K \alpha$ in detriment of hChoK $\beta$ is explained by a less flexible hChoK $\beta$ Cho binding site that does not allow for better accommodation of the inhibitors; (c) compound 5a elucidated that the dimeric form of hChoKa1 presented allosteric properties and in particular negative cooperativity that was characterized by chemical communication between the active sites of both monomers; and (d) the Cho binding site is more exploitable for drug design and is responsible for the improved affinity of inhibitors. In addition, the majority of the compounds interact with this binding site rather than the 
ATP binding site. Compound 9a allowed the discovery of a new exploitable binding site that was adjacent to the Cho binding site; and surprisingly, TCD-717 was located bound near the surface of the enzyme and not in the active site as designed. This binding mode blocks hChoKa1 binding to Cho and can also block phosphorylation of $\mathrm{Y} 333$ that can lead to a decrease in the enzymatic activity of hChoKa.

Although the successful development of Choo inhibitors for the treatment of cancer has resulted in one molecule reaching clinical trials, an approved ChoK $\alpha$ inhibitor for the treatment of specific types of cancer is still lacking. Because of the similarities between human ChoK $\alpha$ and orthologs from pathogens, the vast number of Cho $\alpha$ inhibitors should be evaluated, to fulfil the promise of targeting this important enzyme for the treatment of cancer and infectious diseases. Hopefully, the coming years will prove these compounds as useful therapeutics.

\section{REFERENCES}

1. World Health Organization. https://www.who.int/news-room/fact-sheets/detail/cancer. Accessed December 5, 2019.

2. Bhakoo KK, Williams SR, Florian $\mathrm{CL}$, Land $\mathrm{H}$, Noble MD. Immortalization and transformation are associated with specific alterations in choline metabolism. Cancer Res. 1996;56(20):46304635.

3. Glunde K, Bhujwalla ZM, Ronen SM. Choline metabolism in malignant transformation. Nat Rev Cancer. 2011;11(12):835-848.

4. Arlauckas SP, Browning EA, Poptani H, Delikatny EJ. Imaging of cancer lipid metabolism in response to therapy. NMR Biomed. 2019;32(10):e4070.

5. Iorio E, Caramujo MJ, Cecchetti S, et al. Key Players in Choline Metabolic Reprograming in Triple-Negative Breast Cancer. Front Oncol. 2016;6:205.

6. Bagnoli M, Granata A, Nicoletti R, et al. Choline Metabolism Alteration: A Focus on Ovarian Cancer. Front Oncol. 2016;6:153.

7. de Jong IJ, Pruim J, Elsinga PH, Vaalburg W, Mensink HJ. 11C-choline positron emission tomography for the evaluation after treatment of localized prostate cancer. Eur Urol. 2003;44(1):32-38; discussion 38-39.

8. Utriainen $\mathrm{M}$, Komu $\mathrm{M}$, Vuorinen $\mathrm{V}$, et al. Evaluation of brain tumor metabolism with [11C]choline PET and 1H-MRS. J Neurooncol. 2003;62(3):329-338.

9. Wehrl HF, Schwab J, Hasenbach K, et al. Multimodal elucidation of choline metabolism in a murine glioma model using magnetic resonance spectroscopy and 11C-choline positron emission tomography. Cancer Res. 2013;73(5):1470-1480.

10. Huang Z, Rui J, Li X, Meng X, Liu Q. Use of ${ }^{11} \mathrm{C}$-Choline positron emission tomography/computed tomography to investigate the mechanism of choline metabolism in lung cancer. Mol Med Rep. 2015;11(5):3285-3290.

11. Glunde K, Penet MF, Jiang L, Jacobs MA, Bhujwalla ZM. Choline metabolism-based molecular diagnosis of cancer: an update. Expert Rev Mol Diagn. 2015;15(6):735-747. 
12. Billah MM, Anthes JC. The regulation and cellular functions of phosphatidylcholine hydrolysis. Biochem J. 1990;269(2):281-291.

13. Exton JH. Phosphatidylcholine breakdown and signal transduction. Biochim Biophys Acta. 1994;1212(1):26-42.

14. Gibellini F, Smith TK. The Kennedy pathway--De novo synthesis of phosphatidylethanolamine and phosphatidylcholine. IUBMB Life. 2010;62(6):414-428.

15. McMaster CR. From yeast to humans - roles of the Kennedy pathway for phosphatidylcholine synthesis. FEBS Lett. 2018;592(8):1256-1272.

16. Millington WR, Wurtman RJ. Choline administration elevates brain phosphorylcholine concentrations. J Neurochem. 1982;38(6):1748-1752.

17. Glunde K, Shah T, Winnard PT, et al. Hypoxia regulates choline kinase expression through hypoxia-inducible factor-1 alpha signaling in a human prostate cancer model. Cancer Res. 2008;68(1):172-180.

18. Pelech SL, Vance DE. Regulation of phosphatidylcholine biosynthesis. Biochim Biophys Acta. 1984;779(2):217-251.

19. Uchida T. Stimulation of phospholipid synthesis in HeLa cells by epidermal growth factor and insulin: activation of choline kinase and glycerophosphate acyltransferase. Biochim Biophys Acta. 1996;1304(2):89-104.

20. Ko KW, Cook HW, Vance DE. Reduction of phosphatidylcholine turnover in a Nb 2 lymphoma cell line after prolactin treatment. A novel mechanism for control of phosphatidylcholine levels in cells. J Biol Chem. 1986;261(17):7846-7852.

21. Malito E, Sekulic N, Too WC, Konrad M, Lavie A. Elucidation of human choline kinase crystal structures in complex with the products ADP or phosphocholine. J Mol Biol. 2006;364(2):136151.

22. Hudson CS, Knegtel RM, Brown K, Charlton PA, Pollard JR. Kinetic and mechanistic characterisation of Choline Kinase- $\alpha$. Biochim Biophys Acta. 2013;1834(6):1107-1116.

23. Gallego-Ortega D, Ramirez de Molina A, Ramos MA, et al. Differential role of human choline kinase alpha and beta enzymes in lipid metabolism: implications in cancer onset and treatment. PLoS One. 2009;4(11):e7819.

24. Gallego-Ortega D, Gómez del Pulgar T, Valdés-Mora F, Cebrián A, Lacal JC. Involvement of human choline kinase alpha and beta in carcinogenesis: a different role in lipid metabolism and biological functions. Adv Enzyme Regul. 2011;51(1):183-194.

25. Ramírez de Molina A, Rodríguez-González A, Gutiérrez R, et al. Overexpression of choline kinase is a frequent feature in human tumor-derived cell lines and in lung, prostate, and colorectal human cancers. Biochem Biophys Res Commun. 2002;296(3):580-583.

26. Ramírez de Molina A, Gutiérrez R, Ramos MA, et al. Increased choline kinase activity in human breast carcinomas: clinical evidence for a potential novel antitumor strategy. Oncogene. 2002;21(27):4317-4322.

27. Shah T, Wildes F, Penet MF, et al. Choline kinase overexpression increases invasiveness and drug resistance of human breast cancer cells. NMR Biomed. 2010;23(6):633-642.

28. Arlauckas SP, Popov AV, Delikatny EJ. Choline kinase alpha-Putting the ChoK-hold on tumor metabolism. Prog Lipid Res. 2016;63:28-40.

29. Chang CC, Few LL, Konrad M, See Too WC. Phosphorylation of Human Choline Kinase Beta by Protein Kinase A: Its Impact on Activity and Inhibition. PLoS One. 2016;11(5):e0154702.

30. Chen $\mathrm{X}$, Qiu $\mathrm{H}$, Wang $\mathrm{C}$, et al. Molecular structure and differential function of choline kinases $\mathrm{CHK} \alpha$ and $\mathrm{CHK} \beta$ in musculoskeletal system and cancer. Cytokine Growth Factor Rev. 2017;33:65-72.

31. Hernández-Alcoceba R, Saniger L, Campos J, et al. Choline kinase inhibitors as a novel approach for antiproliferative drug design. Oncogene. 1997;15(19):2289-2301.

32. Cheng M, Bhujwalla ZM, Glunde K. Targeting Phospholipid Metabolism in Cancer. Front Oncol. 2016;6:266.

33. Hernández-Alcoceba R, Fernández F, Lacal JC. In vivo antitumor activity of choline kinase inhibitors: a novel target for anticancer drug discovery. Cancer Res. 1999;59(13):3112-3118. 
34. Cannon JG, Lee TM, Nyanda AM, Bhattacharyya B, Long JP. Structure-activity relationship studies in the hemicholinium ('HC-3') series. Drug Des Deliv. 1987;1(3):209-218.

35. Cannon JG. Structure-activity aspects of hemicholinium-3 (HC-3) and its analogs and congeners. Med Res Rev. 1994;14(5):505-531.

36. Rodríguez-González A, Ramírez de Molina A, Fernández F, et al. Inhibition of choline kinase as a specific cytotoxic strategy in oncogene-transformed cells. Oncogene. 2003;22(55):88038812.

37. Sánchez-Martín R, Campos JM, Conejo-García A, et al. Symmetrical bis-quinolinium compounds: new human choline kinase inhibitors with antiproliferative activity against the HT29 cell line. J Med Chem. 2005;48(9):3354-3363.

38. Lacal JC, Campos JM. Preclinical characterization of RSM-932A, a novel anticancer drug targeting the human choline kinase alpha, an enzyme involved in increased lipid metabolism of cancer cells. Mol Cancer Ther. 2015;14(1):31-39.

39. Study of Intravenous TCD-717 in Patients With Advanced Solid Tumors. ClinicalTrials.gov Identifier: NCT01215864. http://clinicaltrials.gov/ct2/show/NCT01215864.

40. Hong BS, Allali-Hassani A, Tempel W, et al. Crystal structures of human choline kinase isoforms in complex with hemicholinium-3: single amino acid near the active site influences inhibitor sensitivity. J Biol Chem. 2010;285(21):16330-16340.

41. Zimmerman T, Moneriz C, Diez A, et al. Antiplasmodial activity and mechanism of action of RSM-932A, a promising synergistic inhibitor of Plasmodium falciparum choline kinase. Antimicrob Agents Chemother. 2013;57(12):5878-5888.

42. Rubio-Ruiz B, Castillo-Acosta VM, Pérez-Moreno G, et al. In vitro antiplasmodial and cytotoxic activities of asymmetrical pyridinium derivatives. Eur J Med Chem. 2014;85:289-292.

43. Serrán-Aguilera L, Denton H, Rubio-Ruiz B, et al. Plasmodium falciparum Choline Kinase Inhibition Leads to a Major Decrease in Phosphatidylethanolamine Causing Parasite Death. Sci Rep. 2016;6:33189.

44. Bernard NJ. Rheumatoid arthritis: Choline kinase--more than a cancer therapy target? Nat Rev Rheumatol. 2014;10(12):699.

45. Conejo-García A, Báñez-Coronel M, Sánchez-Martín RM, et al. Influence of the linker in bispyridium compounds on the inhibition of human choline kinase. J Med Chem. 2004;47(22):5433-5440.

46. Conejo-García A, Campos J, Sánchez RM, et al. Choline kinase inhibitory effect and antiproliferative activity of new 1,1',1"-(benzene-1,3,5-triylmethylene)tris[4[(disubstituted)amino]pyridinium] tribromides. Eur J Med Chem. 2003;38(1):109-116.

47. Campos J, Núñez MC, Conejo-García A, et al. QSAR-derived choline kinase inhibitors: how rational can antiproliferative drug design be? Curr Med Chem. 2003;10(13):1095-1112.

48. Conejo-García A, Campos JM, Sánchez-Martín RM, Gallo MA, Espinosa A. Bispyridinium cyclophanes: novel templates for human choline kinase inhibitors. J Med Chem. 2003;46(17):3754-3757.

49. Conejo-García A, Campos JM, Entrena A, Sánchez-Martín RM, Gallo MA, Espinosa A. Conformational dynamics of a bispyridinium cyclophane. J Org Chem. 2003;68(22):8697-8699.

50. Conejo-García A, Entrena A, Campos JM, Sánchez-Martín RM, Gallo MA, Espinosa A. Towards a model for the inhibition of choline kinase by a new type of inhibitor. Eur $J$ Med Chem. 2005;40(3):315-319.

51. Conejo-García A, Campos J, Eder C, Entrena A, Gallo MA, Espinosa A. Synthesis and NMR studies on a C3-symmetrical triquinolina triscationic bicyclophane. J Org Chem. 2005;70(14):5748-5751.

52. Campos JM, Sánchez-Martín RM, Conejo-García A, Entrena A, Gallo MA, Espinosa A. (Q)SAR studies to design new human choline kinase inhibitors as antiproliferative drugs. Curr Med Chem. 2006;13(11):1231-1248.

53. Janardhan S, Srivani P, Sastry GN. Choline kinase: an important target for cancer. Curr Med Chem. 2006;13(10):1169-1186. 
54. Campos J, Núñez C, Díaz JJ, Sánchez RM, Gallo MA, Espinosa A. Anticancer bisquaternary heterocyclic compounds: a ras-ional design. Farmaco. 2003;58(3):221-229.

55. Gómez-Pérez V, McSorley T, See Too WC, Konrad M, Campos JM. Novel 4-amino bis-pyridinium and bis-quinolinium derivatives as choline kinase inhibitors with antiproliferative activity against the human breast cancer SKBR-3 cell line. ChemMedChem. 2012;7(4):663-669.

56. Castro-Navas FF, Schiaffino-Ortega S, Carrasco-Jimenez MP, et al. New more polar symmetrical bipyridinic compounds: new strategy for the inhibition of choline kinase a1. Future Med Chem. 2015;7(4):417-436.

57. Schiaffino-Ortega S, Baglioni E, Mariotto E, et al. Design, synthesis, crystallization and biological evaluation of new symmetrical biscationic compounds as selective inhibitors of human Choline Kinase $\alpha 1$ (ChoKa1). Sci Rep. 2016;6:23793.

58. Mariotto E, Bortolozzi R, Volpin I, et al. EB-3D a novel choline kinase inhibitor induces deregulation of the AMPK-mTOR pathway and apoptosis in leukemia T-cells. Biochem Pharmacol. 2018;155:213-223.

59. Mariotto E, Viola G, Ronca R, et al. Choline Kinase Alpha Inhibition by EB-3D Triggers Cellular Senescence, Reduces Tumor Growth and Metastatic Dissemination in Breast Cancer. Cancers (Basel). 2018;10(10).

60. Sola-Leyva A, López-Cara LC, Ríos-Marco P, Ríos A, Marco C, Carrasco-Jiménez MP. Choline kinase inhibitors EB-3D and EB-3P interferes with lipid homeostasis in HepG2 cells. Sci Rep. 2019;9(1):5109.

61. Trousil S, Carroll L, Kalusa A, Aberg O, Kaliszczak M, Aboagye EO. Design of symmetrical and nonsymmetrical. Medchemcomm. 2013;2013(4):693-696.

62. Trousil S, Kaliszczak M, Schug Z, et al. The novel choline kinase inhibitor ICL-CCIC-0019 reprograms cellular metabolism and inhibits cancer cell growth. Oncotarget. 2016;7(24):37103-37120.

63. Martín-Cantalejo Y, Sáez B, Monterde MI, Murillo MT, Braña MF. Synthesis and biological activity of new bispyridinium salts of 4,4'-bispyridyl-5,5'-perfluoroalkyl-2,2'-bisoxazoles. Eur J Med Chem. 2011;46(11):5662-5667.

64. Arlauckas SP, Popov AV, Delikatny EJ. Direct inhibition of choline kinase by a near-infrared fluorescent carbocyanine. Mol Cancer Ther. 2014;13(9):2149-2158.

65. Arlauckas SP, Kumar M, Popov AV, Poptani H, Delikatny EJ. Near infrared fluorescent imaging of choline kinase alpha expression and inhibition in breast tumors. Oncotarget. 2017;8(10):16518-16530.

66. Estévez-Braun A, Ravelo AG, Pérez-Sacau E, Lacal JC. A new family of choline kinase inhibitors with antiproliferative and antitumor activity derived from natural products. Clin Trans/ Oncol. 2015;17(1):74-84.

67. Peisach $D$, Gee $P$, Kent $C, X u Z$. The crystal structure of choline kinase reveals a eukaryotic protein kinase fold. Structure. 2003;11(6):703-713.

68. Milanese L, Espinosa A, Campos JM, Gallo MA, Entrena A. Insight into the inhibition of human choline kinase: homology modeling and molecular dynamics simulations. ChemMedChem. 2006;1(11):1216-1228.

69. LoGrasso PV, Frantz B, Rolando AM, O'Keefe SJ, Hermes JD, O'Neill EA. Kinetic mechanism for p38 MAP kinase. Biochemistry. 1997;36(34):10422-10427.

70. Rubio-Ruiz B, Conejo-Garcia A, Rios-Marco P, et al. Design, synthesis, theoretical calculations and biological evaluation of new non-symmetrical choline kinase inhibitors. European Journal of Medicinal Chemistry. 2012;50:154-162.

71. Rubio-Ruiz B, Conejo-Garcia A, Gallo MA, Espinosa A, Entrena A. 1H and 13C NMR spectral assignments of pyridinium salts linked to a N-9 or N-3 adenine moiety. Magnetic Resonance in Chemistry. 2012;50(6):466-469.

72. Sahun-Roncero M, Rubio-Ruiz B, Saladino G, et al. The Mechanism of Allosteric Coupling in Choline Kinase1 Revealed by the Action of a Rationally Designed Inhibitor. Angewandte Chemie-International Edition. 2013;52(17):4582-4586. 
73. Sahun-Roncero M, Rubio-Ruiz B, Conejo-Garcia A, Velazquez-Campoy A, Entrena A, HurtadoGuerrero R. Determination of Potential Scaffolds for Human Choline Kinase 1 by Chemical Deconvolution Studies. Chembiochem. 2013;14(11):1291-1295.

74. Rubio-Ruiz B, Ramos-Torrecillas J, Capitan-Canadas F, et al. Antiproliferative Activity, Cell Cycle, and Apoptosis Studies of a Series of 6-Substituted 9H-Purin-9-yl-pyridinium Derivatives on a Human Cervical Carcinoma Cell Line. Chemmedchem. 2013;8(8):1266-1269.

75. Rubio-Ruiz B, Rios-Marco P, Carrasco-Jiménez MP, et al. Choline kinase inhibition and docking studies of a series of 6-(benzylthio)-9H-purin-9-yl-pyridinium derivatives. Medicinal Chemistry Research. 2017;26:2809-2815.

76. Schiaffino-Ortega S, López-Cara LC, Ríos-Marco P, et al. New non-symmetrical choline kinase inhibitors. Bioorg Med Chem. 2013;21(22):7146-7154.

77. Rubio-Ruiz B, Figuerola-Conchas A, Ramos-Torrecilas J, et al. Discovery of a New Binding Site on Human Choline Kinase alpha 1: Design, Synthesis, Crystallographic Studies, and Biological Evaluation of Asymmetrical Bispyridinium Derivatives. Journal of Medicinal Chemistry. 2014;57(2):507-515.

78. Serrán-Aguilera L, Nuti R, López-Cara LC, et al. Pharmacophore-Based Virtual Screening to Discover New Active Compounds for Human Choline Kinase a1. Mol Inform. 2015;34(6-7):458466.

79. Clem BF, Clem AL, Yalcin A, et al. A novel small molecule antagonist of choline kinase- $\alpha$ that simultaneously suppresses MAPK and PI3K/AKT signaling. Oncogene. 2011;30(30):3370-3380.

80. Zech SG, Kohlmann A, Zhou T, et al. Novel Small Molecule Inhibitors of Choline Kinase Identified by Fragment-Based Drug Discovery. J Med Chem. 2016;59(2):671-686.

81. Falcon SC, Hudson CS, Huang Y, et al. A non-catalytic role of choline kinase alpha is important in promoting cancer cell survival. Oncogenesis. 2013;2:e38.

82. Kall SL, Delikatny EJ, Lavie A. Identification of a Unique Inhibitor-Binding Site on Choline Kinase a. Biochemistry. 2018;57(8):1316-1325.

83. Kall SL, Whitlatch K, Smithgall TE, Lavie A. Molecular basis for the interaction between human choline kinase alpha and the SH3 domain of the c-Src tyrosine kinase. Sci Rep. 2019;9(1):17121.

84. Serran-Aguilera L, Nuti R, López-Cara LC, et al. Choline kinase active site provides features for designing versatile inhibitors. Curr Top Med Chem. 2014;14(23):2684-2693.

85. Zimmerman T, Lacal JC, Ibrahim SA. Choline Kinase Emerges as a Promising Drug Target in Gram-Positive Bacteria. Front Microbiol. 2019;6:2146.

\section{BIOSKETCHES}

Belén Rubio-Ruiz obtained her BSc in Pharmaceutical Sciences (2007) and her MCs in Research in Drug Development (2008) at the University of Granada (Spain). After obtaining her PhD degree working with ChoK inhibitors, she joined the group of Prof. Unciti-Broceta at the University of Edinburgh (United Kingdom) funded by the European Commission where her research was focused on the development of prodrugs specifically activated by metal catalysis. In 2018 , she returned to the University of Granada to start her independent career at the Pfizer-UGR-Junta de Andalucía Centre for Genomics and Oncological Research and explore the uses of transition metals for combination therapies.

Lucía Serrán-Aguilera graduated in pharmacy from the University of Granada (Spain) in 2009 and later obtained her doctorate from the same institution in 2015 working on new choline kinase inhibitors. She completed different research placements in Perugia (Italy), Zaragoza (Spain) and Oxford (United 
Kingdom) where she focused on the design, synthesis, biological evaluation and crystallization of new anticancer and antihypoxic drugs. Lucía has recently started as a pharmaceutical inspector and collaborates with the University on projects related to new drug development.

Ramón Hurtado-Guerrero is currently an ARAID researcher (Principal Investigator) at the Institute for Biocomputation and Physics of Complex Systems at the University of Zaragoza (Spain). From 2018, he was a visiting Professor at the Copenhagen Center for Glycomics at the University of Copenhagen (Denmark). He is a glycobiologist who uses protein X-ray crystallography and cryo-EM in combination with enzyme kinetics, calorimetry/SPR and enzyme inhibition to study the structural enzymology of glycosyltransferases (GTs), glycosyl hydrolases and transglycosylases involved in the synthesis, modification and degradation of diverse glycoconjugates, oligo- and polysaccharides.

In particular, his research is focused on unraveling important longstanding questions in the field of protein $\mathrm{O}$ - and $\mathrm{N}$-glycosylation and in the development of inhibitors for fungal diseases. Among other questions, he is engaged with how GTs catalyze hundreds of dissimilar protein substrates, and how the lectin domain of GalNAc-Ts tunes catalysis. He also investigates the molecular recognition of antibodies on antigens Tn and STn for the development of vaccines against cancer.

Finally, he is involved in the structural elucidation of choline kinase inhibitors' inhibition mechanism for the tailored design of molecules to tackle cancer.

Ana Conejo-García obtained her BSc in Pharmaceutical Sciences in 1998 at the University of Granada (Spain). In 2000, she did a research placement at University College London (United Kingdom) under the supervision of Prof. Ganellin. She was awarded her PhD in 2002 from the University of Granada (Summa cum Laude, PhD Extraordinary award) where she worked with ChoK inhibitors as anticancer compounds. In 2003, she joined the group of Prof. Schofield at University of Oxford (United Kingdom) and focused on 2-oxoglutarate dependent oxygenases to identify small-molecule inhibitors of major biomedicinal interest in oxygen sensing, and in the epigenetic regulation of gene expression. She returned to the University of Granada in 2006 as Assistant Professor at the Department of Medicinal and Organic Chemistry. She was promoted to Associate Professor in 2009 and to Full Professor of Organic Chemistry in November 2018.

Her research is mainly focused on studying mechanisms that regulate proliferation, differentiation and cellular apoptosis and the search for new therapeutic strategies based on the rational design of highly selective antitumor agents. 


\section{FIGURE LEGENDS}

Figure 1. Structural modifications carried out on HC-3 that led to bis- and tris-quaternary ammonium salts.

Figure 2. Chemical structure of symmetric biscationic derivatives and natural product $22 \beta$-hydroxytingenone.

Figure 3. Historical chart depicting the ChoK X-ray crystal structures reported up to date. The PDB IDs are indicated for each structure. The crystal structures have been determined for different ChoK orthologs in which $C e, h, P k, P f, C p$ and $S p$ stand for Caenorhabditis elegans, human, Plasmodium knowlesi, Plasmodium falciparum, Cryptosporidium parvum and Streptococcus pneumoniae, respectively. Compounds are numbered according to the main text with the exception of compounds 11 and 37 that keep the original names indicated in the corresponding PBD files. *No publications have been associated with this PDB ID to date.

Figure 4. a) Close-up view of the ATP binding site of the hChoK $\alpha 2$ isoform. b) Cho binding site. Red dashed lines indicate hydrogen bonds.

Figure 5. a) Close-up view of hChoK $\alpha 1$ complexed to HC-3. b) Close-up view of hChoK $\beta$ complexed to pHC-3.

Figure 6. First proposed hChoK catalytic mechanism based on the formation of a ternary complex with ATP, $\mathrm{Mg}^{2+}$ and Cho.

Figure 7. Second proposed mechanism of action for hChoK based on the formation of a phosphorylated intermediate (P-D306).

Figure 8. a) Chemical structure of pyridinium derivatives $\mathbf{5 a}$ and $\mathbf{5 b}$. b) Compound $\mathbf{5 a}$ binds to both ATP and Cho binding sites. c) Residues of the active site that stabilize $\mathbf{5 a}$. Red dashes indicate hydrogen bond interactions.

Figure 9. Chemical structures of non-symmetric pyridinium compounds $\mathbf{6 , 7 , 8 a}$ and $\mathbf{8 b}$.

Figure 10. a) Chemical structure of compounds 9a-9c. b) Close-up view of the crystal structure of hChoK $\alpha 1-9 a$ complex that shows the newly discovery binding pocket adjacent to the Cho binding site, which is formed by residues W248, T252, Y256, Y333, L419, W420, and W423.

Figure 11. a) Pharmacophore model and chemical structures of the three most potent hChoK $\alpha 1$ binders discovered by this pharmacophore-based approach. b) Crystal structure of hChoKa1-12 complex. c) Residues that interact to the two molecules of $\mathbf{1 2}$. Hydrogen bonds are represented by red dashes.

Figure 12. Compound CK37.

Figure 13. a) Chemical structure of compounds 13-15. b) hChoKa-13 X-ray crystal structure. c) Closeup view of $\mathbf{1 3}$ and the residues of Cho binding site that contribute to its stabilization. Red dashes represent hydrogen bond interactions. 
Figure 14. a) Compounds V-11-023907 and V-11-0711. b) Crystal structure of hChoK $\alpha-V-11-023907$ complex. c) Close-up view of the Cho binding site showing the residues that interact with V-11-023907. Red dashes represent hydrogen bonds.

Figure 15. a) TCD-717 binding mode. b) Residues of the binding site that stabilize the compound. c) Close-up view of ATP and Cho binding sites that depict the orientation of ADP and PCho with respect to TCD-717.

Figure 16. a) Chemical structure of compounds 16 and 17 and the $K_{d} s$ values $(\mu \mathrm{M})$ among ChoK orthologs. b) Surface representation of the hChoK $\alpha 1$ colored by sequence conservation: black (100\% identity), grey ( $\approx 50 \%$ identity) and white (no identity). Sequence conservation was inferred by comparison of the amino acid sequences between hChoK $\alpha 1$, PfChoK and CpChoK. The overall structure shows ADP (PDB ID 3G15) and a modelled PCho for illustration purposes. c) Close-up views on ADP (above) and PCho (below) binding sites. 


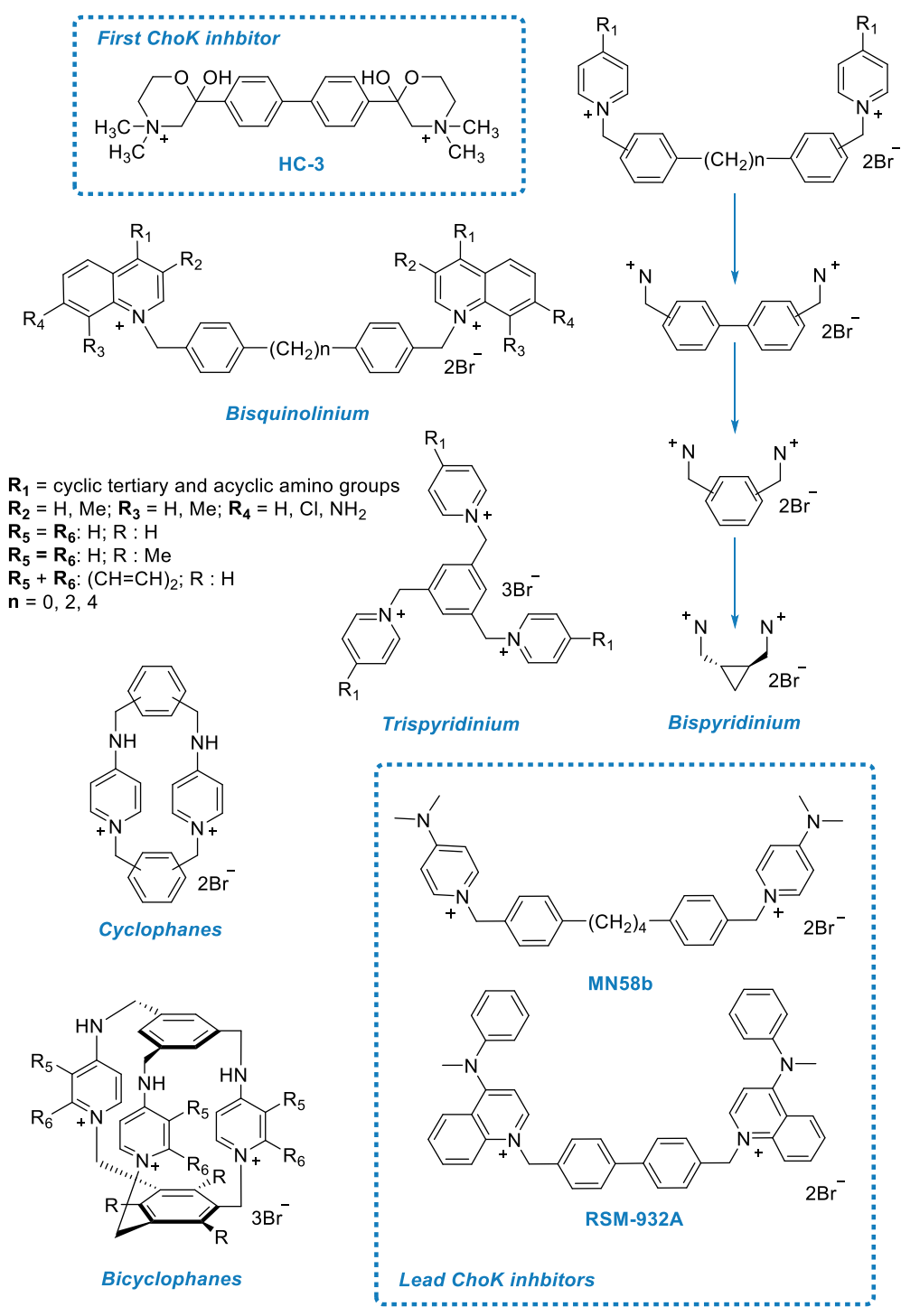

Figure 1. 

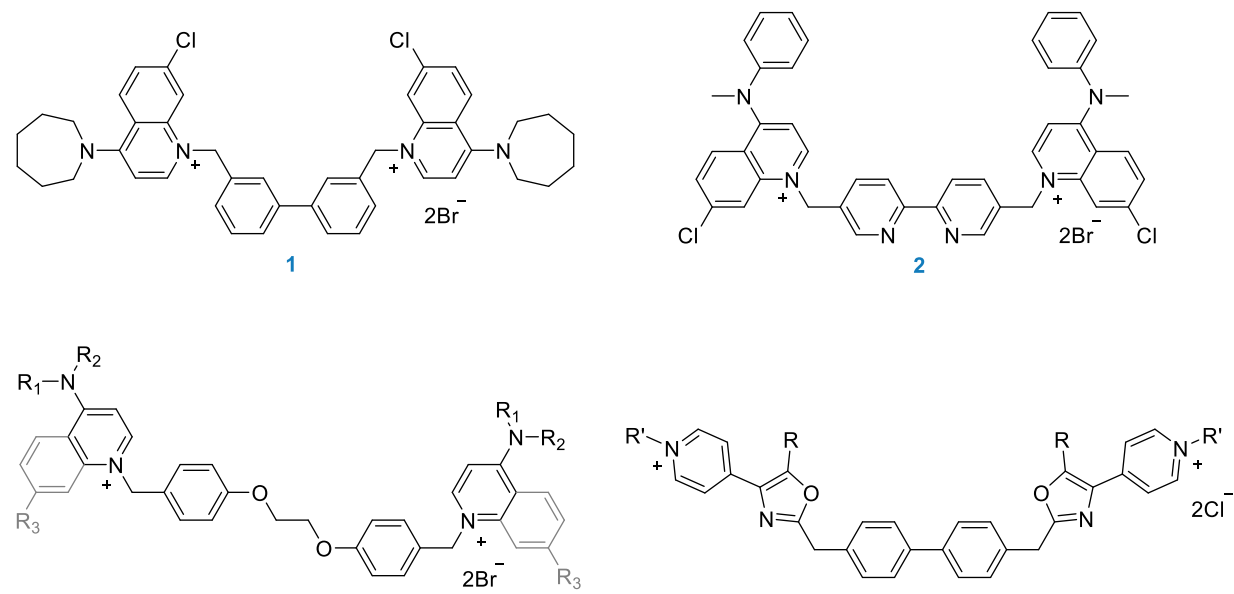

3a: pyridinium, $\mathrm{R}_{1}=\mathrm{R}_{2}=-\mathrm{CH}_{3}$

3b: quinolinium, $\mathrm{R}_{1}+\mathrm{R}_{2}=-\left(\mathrm{CH}_{2}\right)_{4} ; \mathrm{R}_{3}=-\mathrm{Cl}$

4a $\mathrm{R}=\mathrm{CF}_{3} \mathrm{CF}_{2}, \mathrm{R}^{\prime}=\mathrm{CH}_{2}-\mathrm{CH}_{2} \mathrm{OH}$

4b $\mathrm{R}=\mathrm{CF}_{3}, \mathrm{R}^{\prime}=\mathrm{CH}_{2}-\mathrm{CON}\left(\mathrm{CH}_{3}\right)_{2}$<smiles>CN(C)C1=CCN(C=CC[n+]2ccc(N(C)C)cc2)C=C1</smiles>

ICL-CCIC-0019

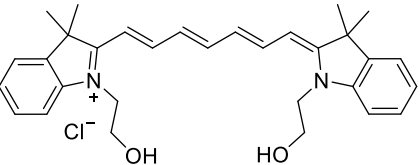

JAS239

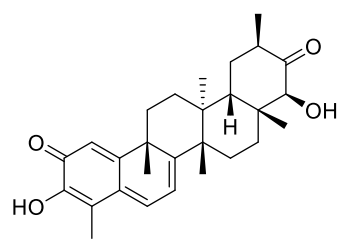

22 -hydroxy-tingenone

Figure 2.

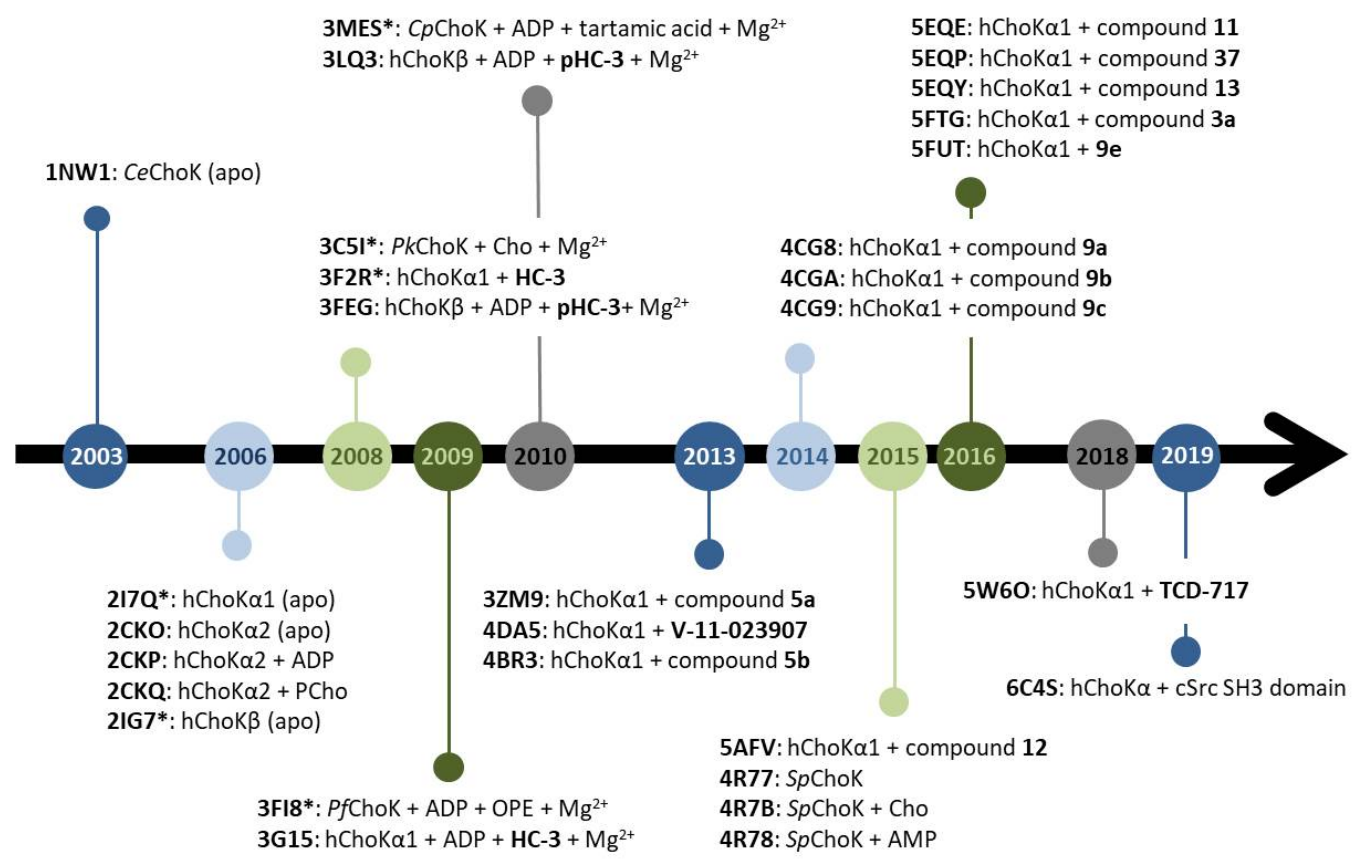

Figure 3. 
a)

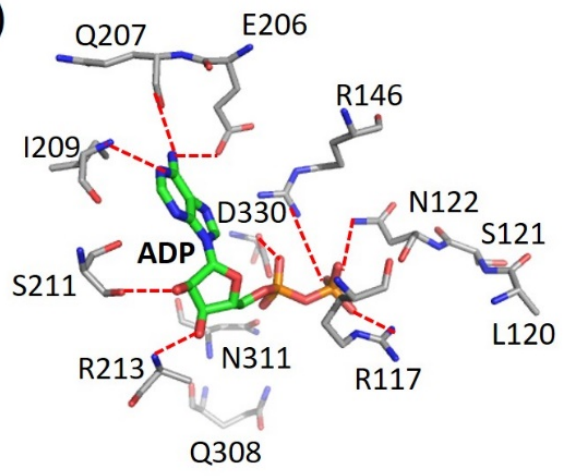

b)

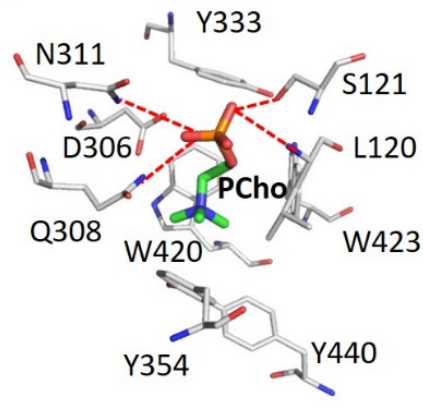

Figure 4.

a)

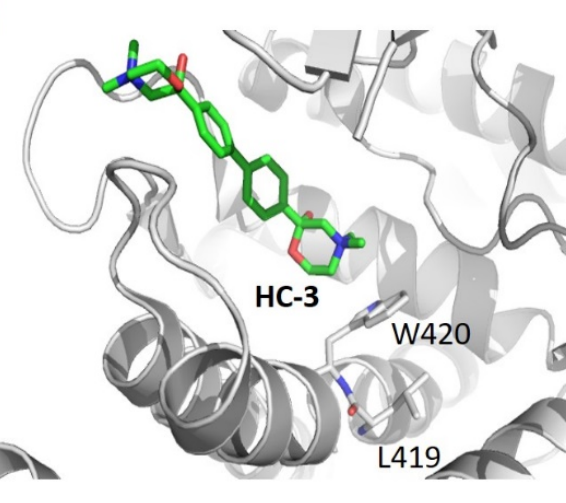

b)

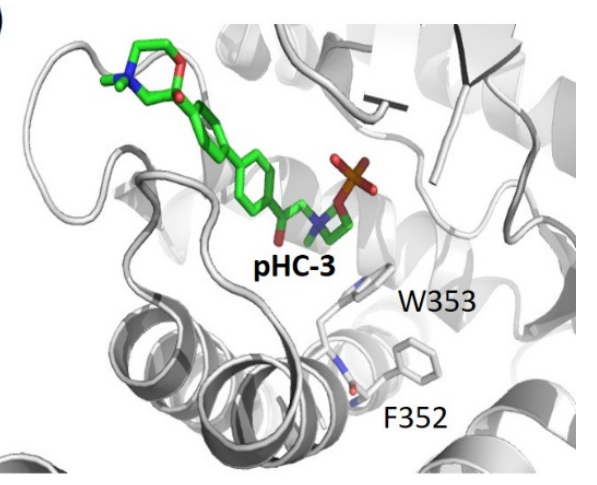

Figure 5.

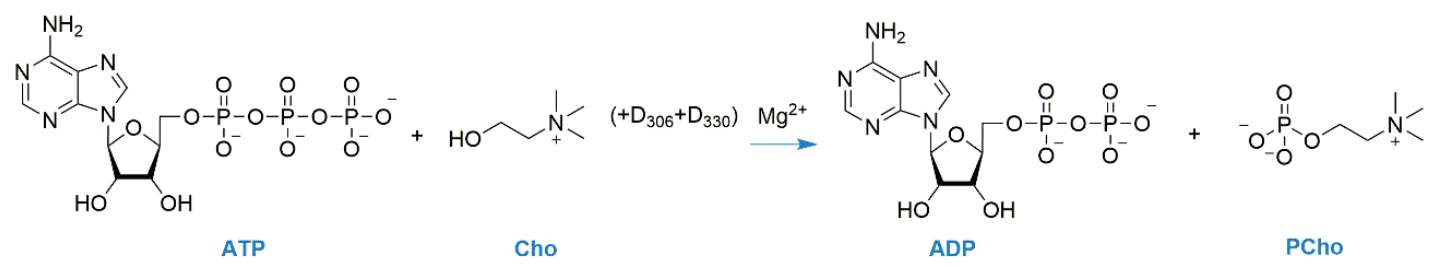

Figure 6.

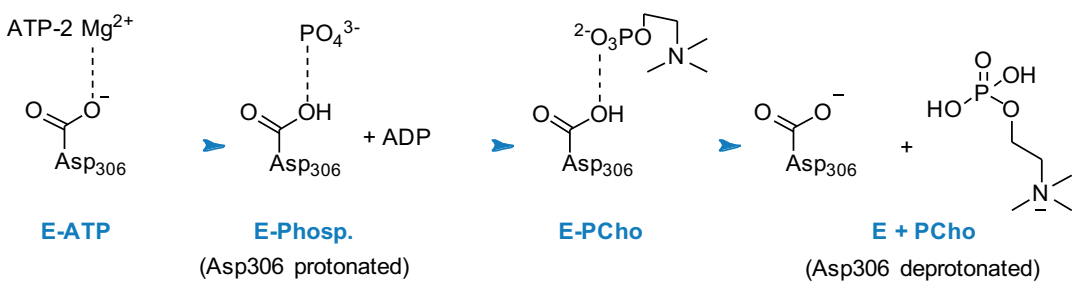

Figure 7. 
a)

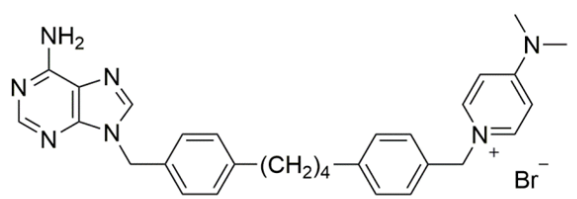

$5 a$

b)

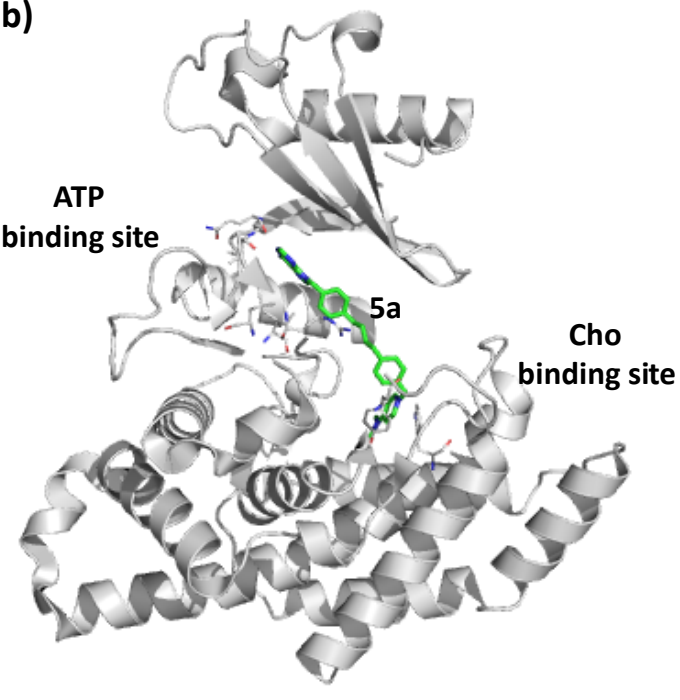

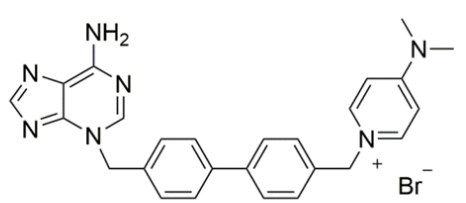

$5 b$

c)

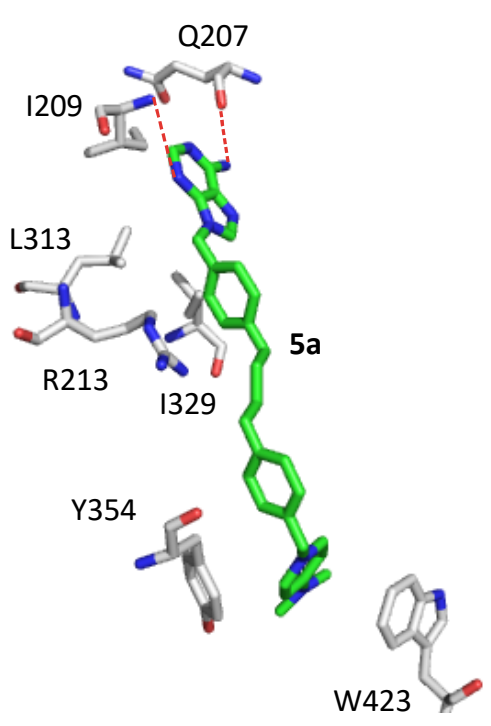

Figure 8.
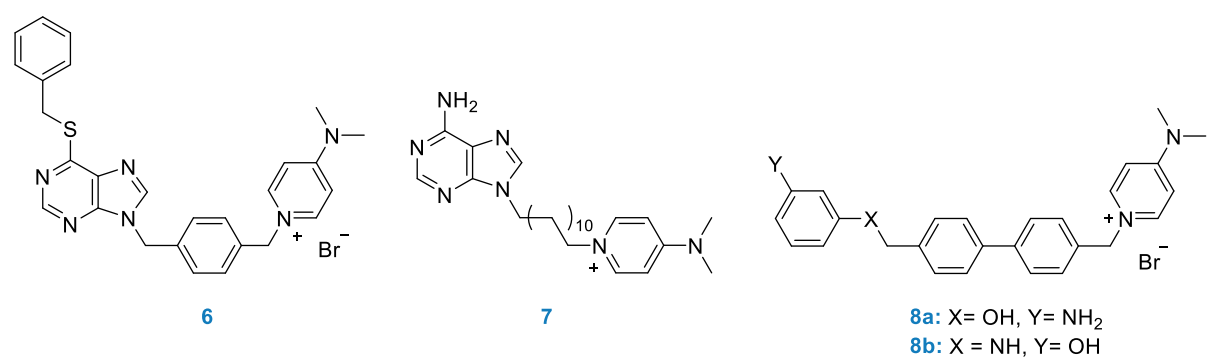

Figure 9. 
a)

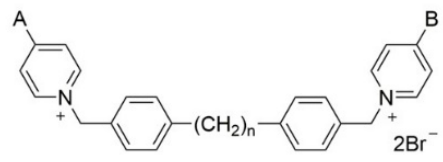

9a $n=4, A=R_{1}, B=R_{3}$

$9 b n=2, A=R_{1}, B=R_{2} \quad 9 d n=0, A=R_{1}, B=R_{2}$ 9c $n=0, A=R_{1}, B=R_{3}$ 9e $n=4, A=R_{1}, B=R_{2}$

$R_{1}=-N^{\prime} \quad R_{2}=-N \quad R_{3}=-N^{\prime}$ b)

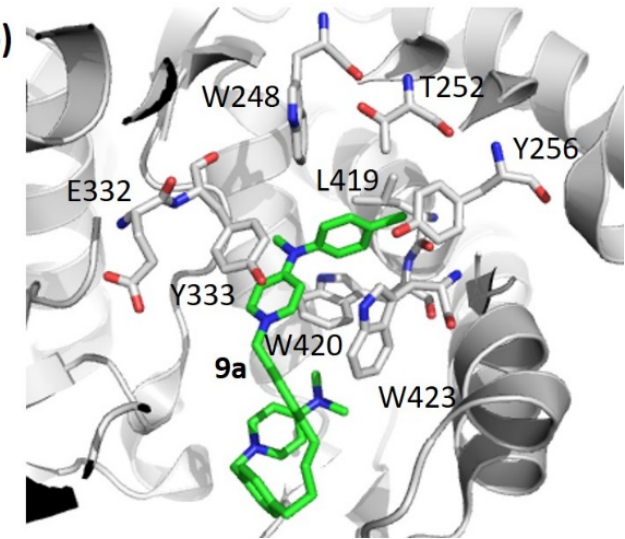

Figure 10.

a)

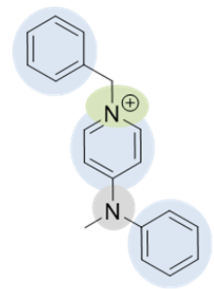

Pharmacophore model

b)

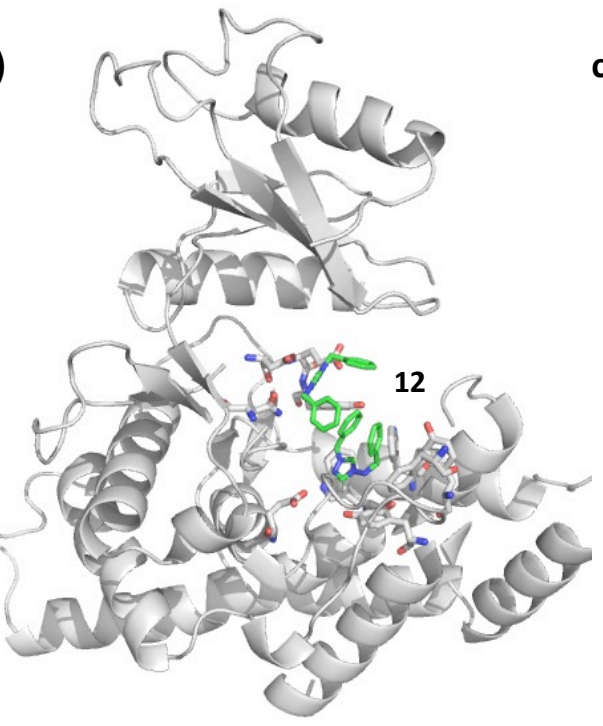

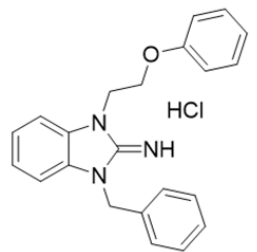

10
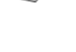

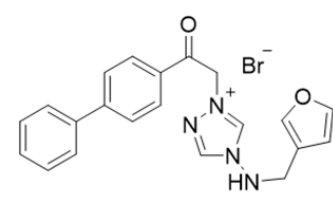

11

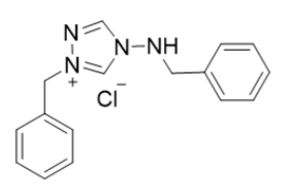

12

c)

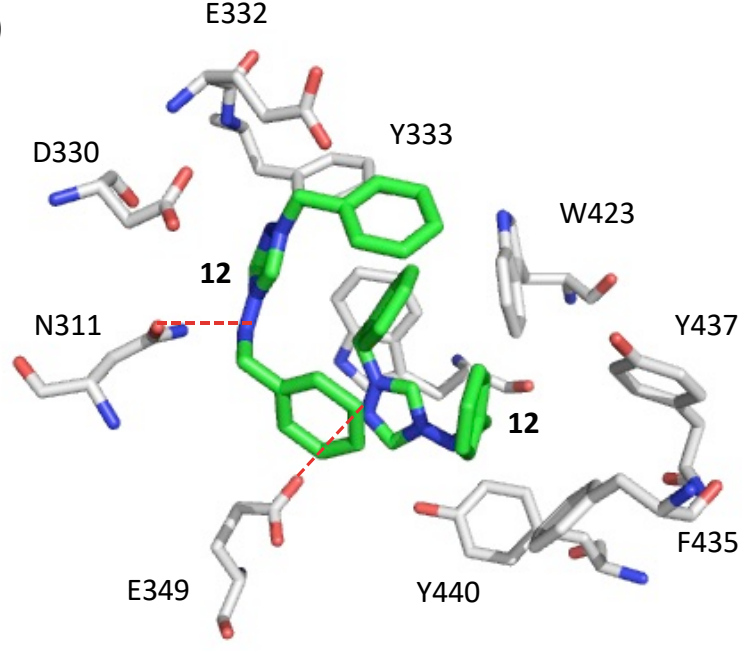

Figure 11. 


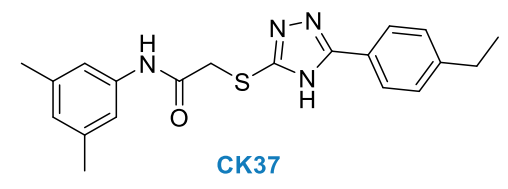

Figure 12.

a)

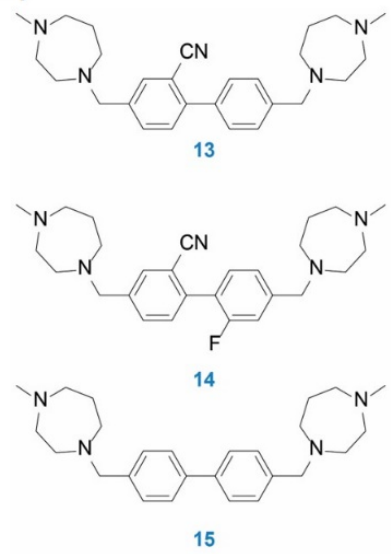

b)

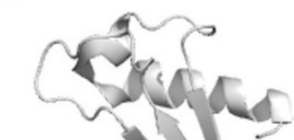

c)
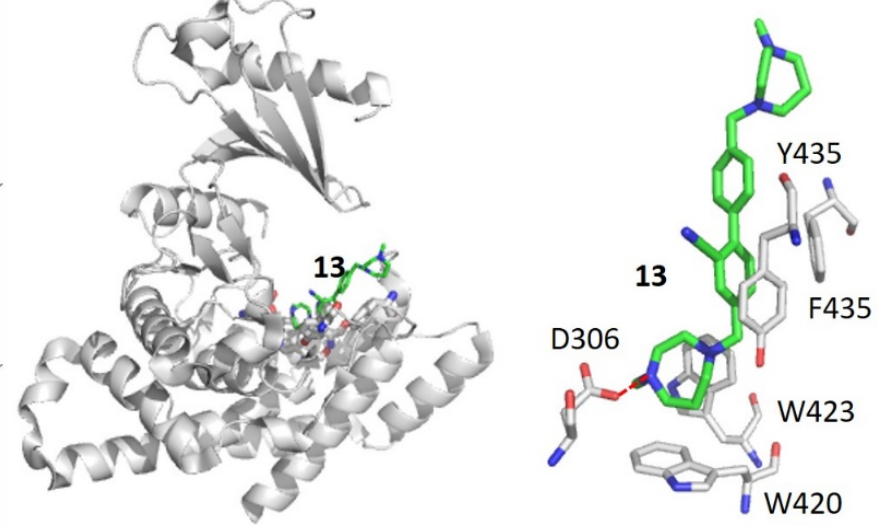

Figure 13.

a)

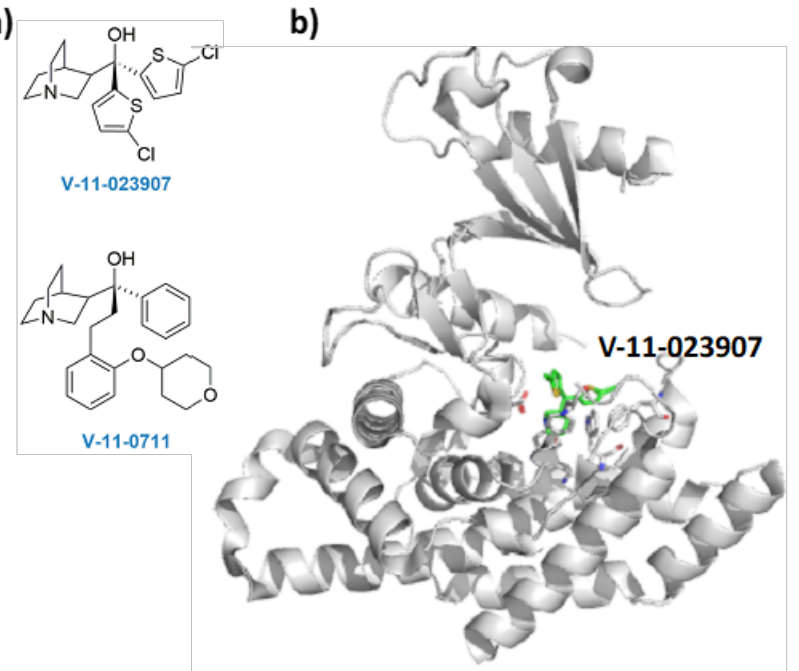

c)

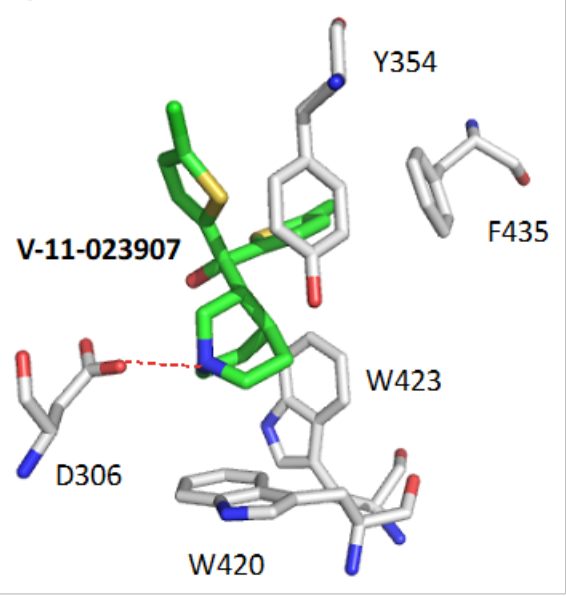

Figure 14. 
a)

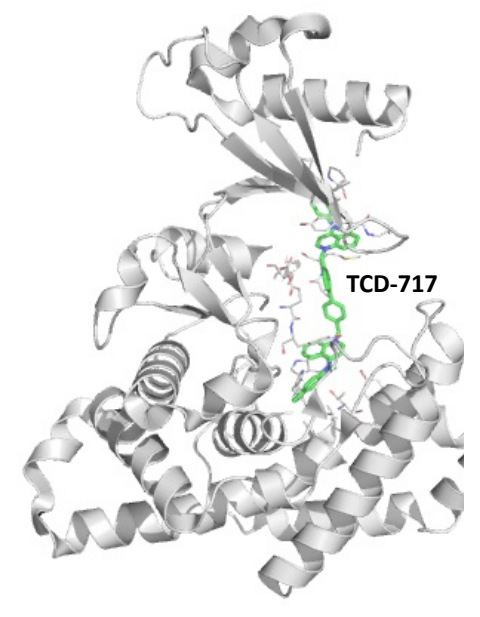

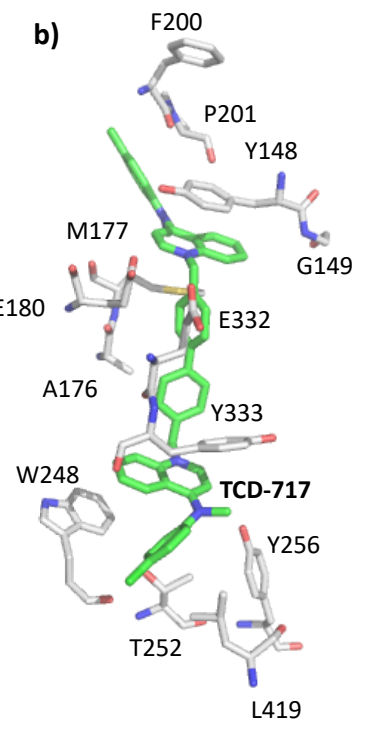

c)

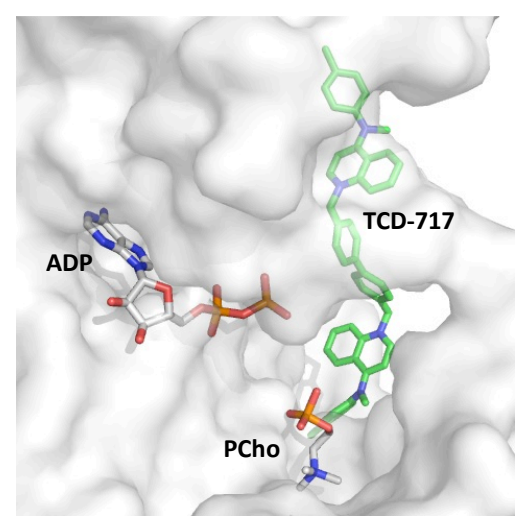

Figure 15.

a)

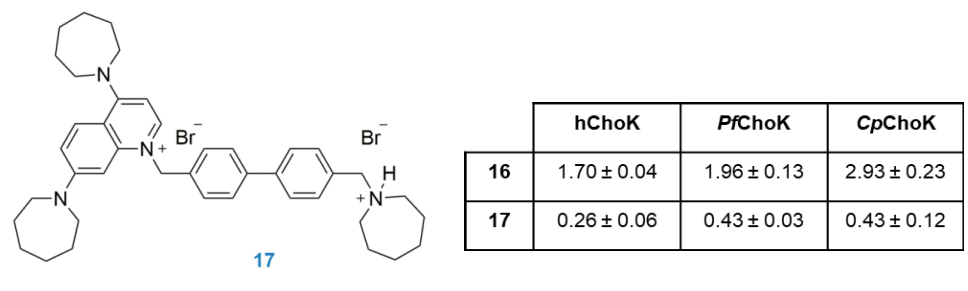

b)

c)
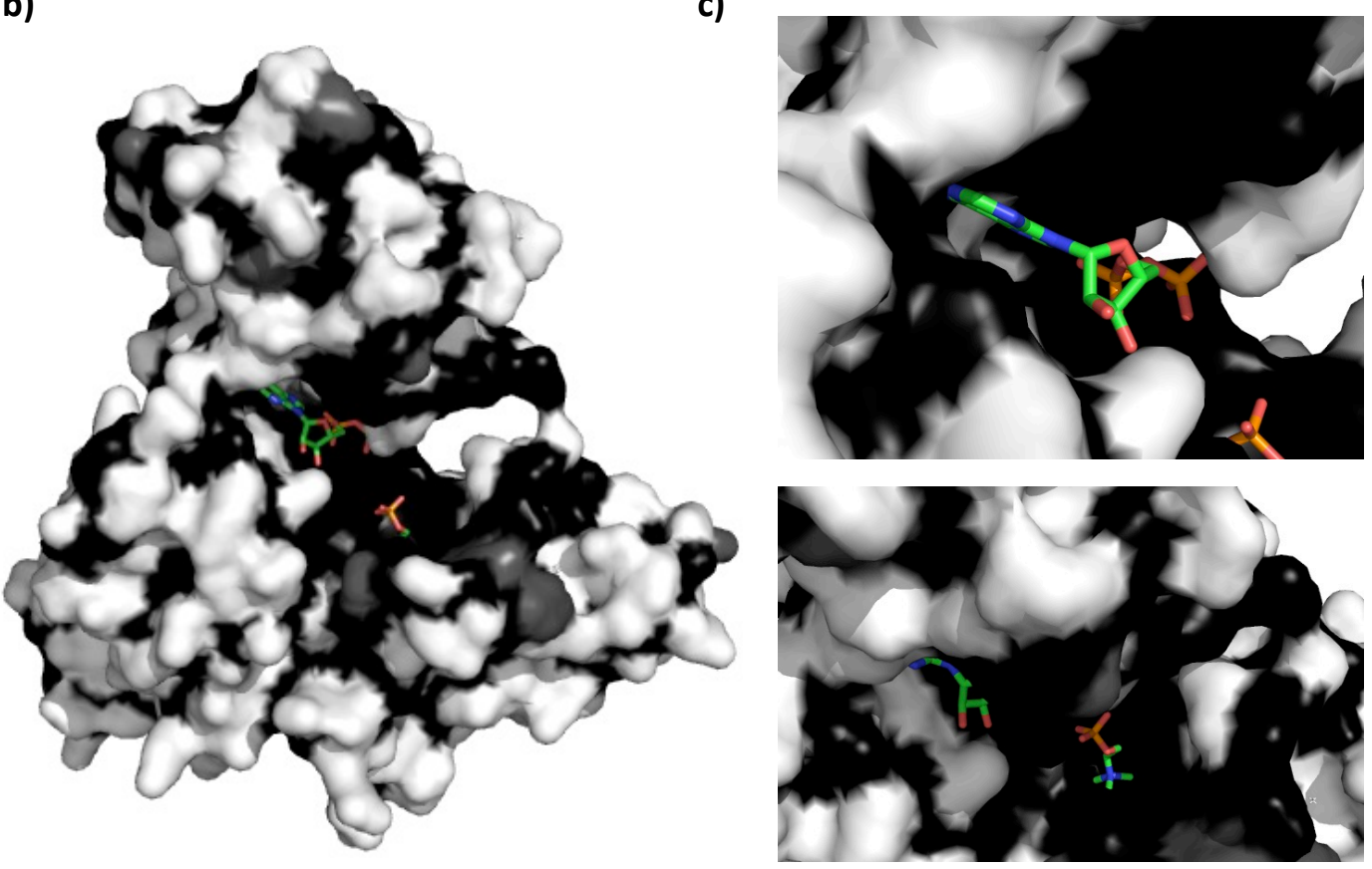

Figure 16. 